\title{
Nanoribbons: From fundamentals to state-of-the-art applications
}

\author{
M. Yagmurcukardes, ${ }^{1, a)}$ F. M. Peeters, ${ }^{2}$ R. T. Senger, ${ }^{1}$ and H. Sahin ${ }^{3, b)}$ \\ ${ }^{1}$ Department of Physics, Izmir Institute of Technology, 35430 Izmir, Turkey \\ ${ }^{2}$ Department of Physics, University of Antwerp, Groenenborgerlaan 171, B-2020 Antwerp, Belgium \\ ${ }^{3}$ Department of Photonics, Izmir Institute of Technology, 35430 Izmir, Turkey
}

(Received 21 September 2016; accepted 19 October 2016; published online 14 November 2016)

\begin{abstract}
Atomically thin nanoribbons (NRs) have been at the forefront of materials science and nanoelectronics in recent years. State-of-the-art research on nanoscale materials has revealed that electronic, magnetic, phononic, and optical properties may differ dramatically when their one-dimensional forms are synthesized. The present article aims to review the recent advances in synthesis techniques and theoretical studies on NRs. The structure of the review is organized as follows: After a brief introduction to low dimensional materials, we review different experimental techniques for the synthesis of graphene nanoribbons (GNRs) with their advantages and disadvantages. In addition, theoretical investigations on width and edge-shape-dependent electronic and magnetic properties, functionalization effects, and quantum transport properties of GNRs are reviewed. We then devote time to the NRs of the transition metal dichalcogenides (TMDs) family. First, various synthesis techniques, E-field-tunable electronic and magnetic properties, and edge-dependent thermoelectric performance of NRs of $\mathrm{MoS}_{2}$ and $\mathrm{WS}_{2}$ are discussed. Then, strongly anisotropic properties, growth-dependent morphology, and the weakly width-dependent bandgap of $\mathrm{ReS}_{2} \mathrm{NRs}$ are summarized. Next we discuss TMDs having a T-phase morphology such as $\mathrm{TiSe}_{2}$ and stable single layer NRs of mono-chalcogenides. Strong edge-type dependence on characteristics of GaS NRs, width-dependent Seebeck coefficient of SnSe NRs, and experimental analysis on the stability of $\mathrm{ZnSe}$ NRs are reviewed. We then focus on the most recently emerging NRs belonging to the class of transition metal trichalcogenides which provide ultra-high electron mobility and highly anisotropic quasi-1D properties. In addition, width-, edge-shape-, and functionalization-dependent electronic and mechanical properties of blackphosphorus, a monoatomic anisotropic material, and studies on NRs of group IV elements (silicene, germanene, and stanene) are reviewed. Observation of substrate-independent quantum well states, edge and width dependent properties, the topological phase of silicene NRs are reviewed. In addition, $\mathrm{H}_{2}$ concentration-dependent transport properties and anisotropic dielectric function of GeNRs and electric field and strain sensitive I-V characteristics of SnNRs are reviewed. We review both experimental and theoretical studies on the NRs of group III-V compounds. While defect and N-termination dependent conductance are highlighted for boron nitride NRs, aluminum nitride NRs are of importance due to their dangling bond, electric field, and strain dependent electronic and magnetic properties. Finally, superlattice structure of NRs of GaN/AIN, Si/Ge, G/BN, and $\mathrm{MoS}_{2} / \mathrm{WS}_{2}$ is reviewed. Published by AIP Publishing.

[http://dx.doi.org/10.1063/1.4966963]
\end{abstract}

\section{TABLE OF CONTENTS}

I. INTRODUCTION $\ldots \ldots \ldots \ldots \ldots \ldots \ldots \ldots \ldots \ldots \ldots$. 2

II. THE RISE OF SINGLE LAYER NRs:

GRAPHENE ........................ 2

A. Synthesis and fabrication techniques....... 2

B. Theoretical Studies on Graphene NRs...... 5

III. NRs OF TRANSITION METAL

DICHALCOGENIDES . .

\footnotetext{
${ }^{\text {a)} E l e c t r o n i c ~ m a i l: ~ m e h m e t y a g m u r c u k a r d e s @ i y t e . e d u . t r ~}$

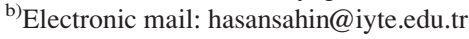

A. NRs of $\mathrm{MoS}_{2} \ldots \ldots \ldots \ldots \ldots \ldots \ldots$

1. Experimental studies on $\mathrm{MoS}_{2} \ldots \ldots \ldots 8$

2. Theoretical studies on $\mathrm{MoS}_{2} \ldots \ldots \ldots .10$

B. NRs of $\mathrm{WS}_{2} \ldots \ldots \ldots \ldots \ldots \ldots \ldots \ldots \ldots \ldots$

C. In-plane-anisotropic NRs: $\operatorname{ReX}_{2} \mathrm{~s} \ldots \ldots \ldots \quad 12$

D. Octahedrally coordinated NRs: $\mathrm{TiSe}_{2} \ldots \ldots, 12$

IV. MONOCHALCOGENIDE NRs ............. 13

A. Gallium monochalcogenides: GaS, GaSe ... 13

B. Mono-selenide NRs: ZnSe and SnSe ...... 13

V. NRs OF TRI-CHALCOGENIDES. ............ 14

A. High electron mobility NRs: $\mathrm{TiS}_{3} \ldots \ldots \ldots 14$

B. Quasi-1D behavior in $\mathrm{ZrS}_{3} \ldots \ldots \ldots \ldots \ldots$

VI. BLACKPHOSPHORUS NRs (bpNRs) ........ 15 
VII. GROUP IV NRs ................ 16

A. GNRs' cousin: Silicene NRs ........... 16

B. NRs of germanene and stanene......... 18

VIII. NRs OF GROUP III-V COMPOUNDS . . . . . . 19

A. 1D form of white graphene: h-boron nitride

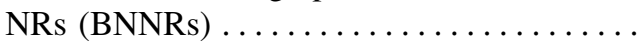

B. h-AlN-NRs: Edge dependent magnetism.... IX. SUPERSTRUCTURES OF NRs............ X. SUMMARY $\ldots \ldots \ldots \ldots \ldots \ldots \ldots \ldots \ldots$

\section{INTRODUCTION}

Over the past thirty years, low-dimensional structures such as wells, wires, and dots have been the focus of interest in materials science. In this material class, confinement of electrons, holes, and excitations in certain directions leads to dramatic changes in electronic, vibrational, optical, thermal, and chemical properties. Unique properties of low-dimensional crystals stem from quantum effects that emerge when at least one of the three dimensions of the crystal is reduced to a sufficiently small size, generally in the range from 1 to $100 \mathrm{~nm}$.

While two-dimensional (2D) thin crystals limit the physical phenomena into a plane, in a one-dimensional (1D) quantum structure (nanowires, nanotubes, and nanoribbons (NRs)), charge carriers and excitations have only one degree of freedom. These crystal structures have been the focus of interest due to their unique properties such as very high electronic density of states, enhanced exciton binding energy, diameter-dependent bandgap, increased surface scattering for electrons and phonons, and chirality-dependent electronic band structure.

Nanoribbons (NRs), made of single- or few-atom-thick lamellar crystals, are novel forms of 1D nanoscale materials and are ideal systems for investigation of the size and dimensionality dependence of the fundamental properties. After successful synthesis of many 2D monolayer materials, their 1D NR form came into prominence due to their necessity in nanoscale applications. In this context, here we present an extensive review on NRs of recent $2 \mathrm{D}$ materials such as graphene, transition metal dichalcogenides (TMDs) $\left(\mathrm{MoS}_{2}, \mathrm{WS}_{2}, \mathrm{ReS}_{2}\right.$, and $\mathrm{TiSe}_{2}$ ), mono-chalcogenides (GaS, GaSe, ZnSe, and $\mathrm{SnSe}$ ), tri-chalcogenides $\left(\mathrm{TiS}_{3}\right.$ and $\mathrm{ZrS}_{3}$ ), blackphosphorus, group-IV, III-V binary compounds, and superstructures.

\section{THE RISE OF SINGLE LAYER NRS: GRAPHENE}

The first breakthrough in 2D atomic-thick materials started following the successful isolation of graphene from its bulk counterpart, graphite, in 2004. ${ }^{1}$ Graphene, which is simply an atomic-thin sheet of hexagonally arranged $\mathrm{C}$ atoms, has many unique physical properties such as being the thinnest but mechanically the strongest and stiffest material. $^{2}$ In addition, graphene is not only mechanically but also electronically a unique material. It has been demonstrated that linear crossing of valence and conduction band edges at the Fermi level provides a very high electron mobility of $15 \times 10^{3} \mathrm{~cm}^{2} / \mathrm{V}$ s and quite high thermal conductivity. ${ }^{3,4}$

As a consequence of their hexagonal lattice symmetry, NRs of graphene can form zigzag and armchair shaped edges which directly determine their magnetic and electronic properties. Theory predicted that depending on their edge shape and the width, graphene nanoribbons (GNRs) can possess magnetic metallic or nonmagnetic (NM) semiconducting ground states which was confirmed in a recent experiment. Recent experiments demonstrated that GNRs can be synthesized successfully with controllable structural and electronic properties. $^{5,6}$ Since GNRs provide a rich playground, recent efforts have focused on the synthesis and deeper understanding of these materials.

\section{A. Synthesis and fabrication techniques}

Atomic structure and the resulting characteristic properties of GNRs strongly depend on how the GNR is synthesized. There are three main experimental methods available to fabricate GNRs: (i) cutting graphene along special directions by using lithography, (ii) bottom-up synthesis from precursor molecules, and (iii) unzipping of carbon nanotubes (CNTs).

The bottom-up fabrication technique allows one to synthesize very narrow ribbons with precise edge configurations. One of the first successful syntheses of narrow GNRs was reported by Li et al. They developed a chemical route to produce GNRs having widths below $10 \mathrm{~nm}$ and single NRs with varying widths. ${ }^{5}$ Instead of using lithography which is unable to produce smooth edges, they chemically exfoliated graphite in dichloroethane (DCE) solution of poly(m-phenylenevinylene-co-2,5-dioctoxy-p-phenylenevinylene) (PmPV) by sonication which resulted in GNRs with desired shape and dimensions. For measurement and characterization of GNRs, atomic force microscopy (AFM) was used and GNRs with various widths ranging from $\sim 50 \mathrm{~nm}$ down to sub$10 \mathrm{~nm}$ were observed (see Fig. 1).

An alternative method of producing atomically precise NRs having different topologies and widths, by using surface-assisted coupling of molecular precursors (such as 6,11-dibromo-1,2,3,4tetraphenyltriphenylene monomers), was reported by Cai et $a l .{ }^{6}$ However, study of Vo et al. on narrow armchair GNRs (AGNRs) $\quad(\sim 1 \mathrm{~nm})$ synthesized by a novel bottom-up approach $^{7}$ revealed that the ribbons have a large electronic bandgap of $\sim 1.3 \mathrm{eV}$, a large aspect ratio, and a length of $>100 \mathrm{~nm}$. These large values of bandgap and ribbon length are quite promising for applications in high on-off ratios fieldeffect transistors and photovoltaic devices. Kimouche et al. studied the fabrication of GNRs with atomically well-defined armchair edges. ${ }^{8}$ They reported the synthesis of the narrowest possible GNR having a width of five carbon atoms by using a bottom-up approach technique (see Fig. 2). The evolution of the electronic bandgap as a function of GNRs length was investigated using low-temperature scanning tunneling microscopy. It was found that the synthesized ultra-narrow GNRs (five $\mathrm{C}$-atom wide, $\mathrm{N}=5$ ) display nearly metallic behavior as predicted theoretically. The main point of their study is that the synthesized armchair GNRs can be used as molecular wires exhibiting metallic behavior at room temperature.

In another study, Bai et al. reported a rational approach for the fabrication of GNRs with sub-10 nm width by utilizing chemically synthesized nanowires as the physical protection 

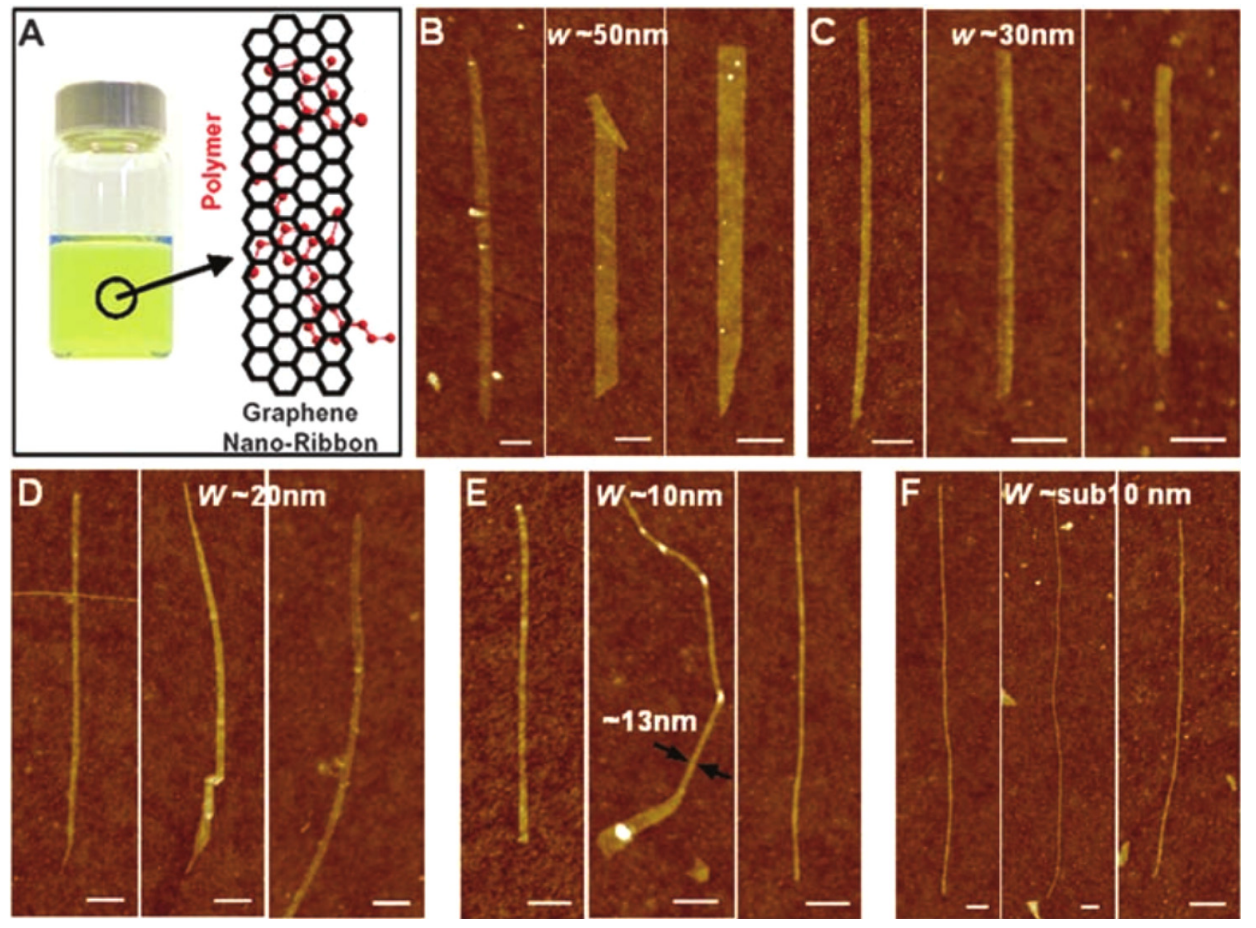

FIG. 1. (a) PmPV/DCE solution with GNRs on the left side and a simulation of GNR with PmPV polymer adsorbed on GNR. (b)-(f) AFM images of GNRs with widths ranging from $50 \mathrm{~nm}$ to sub- $10 \mathrm{~nm}$. All scale bars indicate $100 \mathrm{~nm}$. Reprinted with permission from Li et al., Science 319, 1229 (2008). Copyright 2008 The American Association for the Advancement of Science. mask in oxygen plasma etch. The important point of their study is to fabricate GNRs in a predictable and controllable manner. ${ }^{9}$ It was reported that the width of the fabricated GNRs scales well with the diameter of the nanowire and the etching time. Their fabricated GNRs have widths varying from 6 to

a

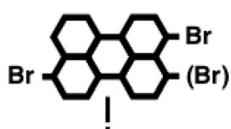

Polymerization at $200^{\circ} \mathrm{C}$ ;

Cyclodehydrogenation at $320^{\circ} \mathrm{C}$ $\downarrow$
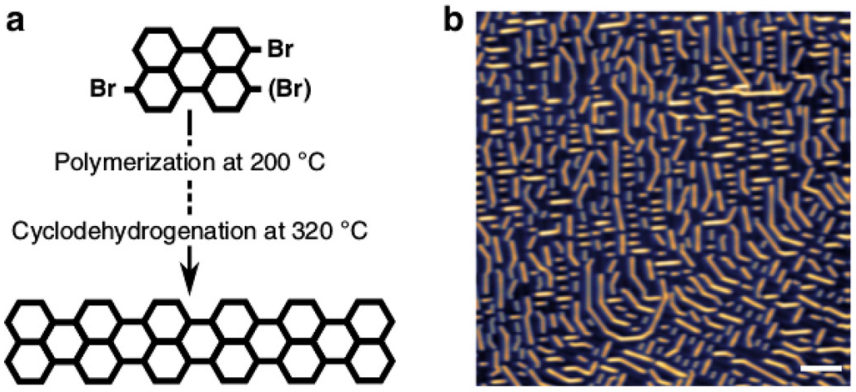

C

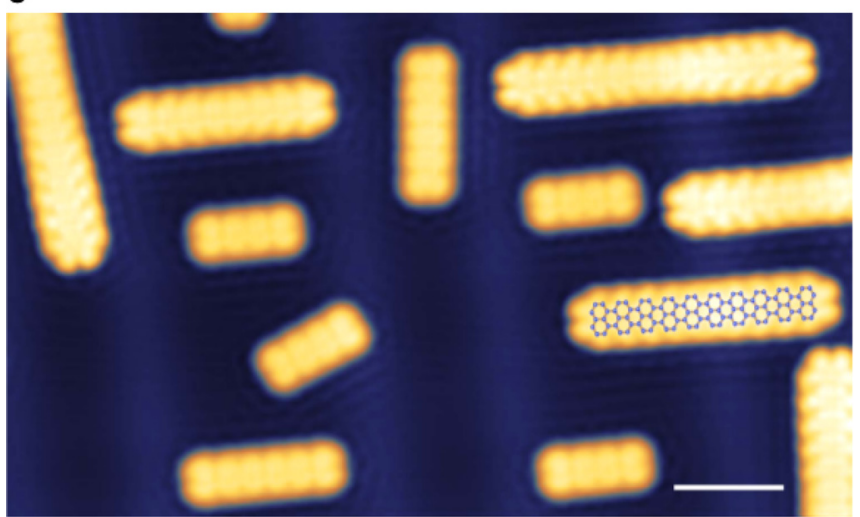

FIG. 2. Bottom-up synthesis of $\mathrm{N}=5$ wide armchair GNRs. (a) Reaction scheme of the polymerization with dibromoperylene (DBP) molecular precursor to atomically well defined $\mathrm{N}=5$ armchair GNRs, (b) STM image after cyclodehydrogenation process at $320^{\circ} \mathrm{C}$ (scale bar, $10 \mathrm{~nm}$ ), and (c) zoomed-in STM topography of different ribbon lengths (scale bar, $2 \mathrm{~nm}$ ). Reprinted with permission from Kimouche et al., Nat. Commun. 6, 10177 (2015). Copyright 2015 Nature Publishing Group.
$30 \mathrm{~nm}$. One of the advantages of the technique used by Bai et al. is that they are able to control the width of the fabricated GNRs by the diameter of the used nanowire mask. Another advantage is the relatively smooth etching edge owing to the uniformity and smoothness of the mask nanowires. Very recently by a similar methodology, Ruffieux et al. reported the synthesis of GNRs with a zigzag edge topology. ${ }^{10}$ They demonstrated a bottom-up synthesis of zigzag graphene NRs (ZGNRs) through surface-assisted polymerization and cyclodehydrogenation of special precursor monomers. Although it was theoretically predicted, the observation of spin-polarized edge states in ZGNRs has not yet been achieved in the experiments. However, by using scanning tunneling spectroscopy (STS) they showed the existence of edge-localized states with large energy splittings in ZGNRs. As mentioned in their study, it is important to control the edge modification of ZGNRs by reducing the ZGNR substrate interaction. Campos et al. fabricated GNRs with sub-10 nm width using anisotropic etching of monolayer graphene by thermally activated nickel (Ni) nanoparticles. ${ }^{11}$ A new catalytic channeling behavior was observed and etched cuts of graphene result in continuously connected geometries. Their Raman spectroscopy and electronic measurements indicate that the quality of graphene does not strongly depend on the etching conditions. Thus, the mentioned method may be a powerful tool to obtain GNRs with well-defined edges.

Atomic force microscopy (AFM) based local anodic oxidation (LAO) lithography has been used to fabricate microand nano-structures on different substrates. It is possible to include the ability to pattern surfaces with nanometer resolution by LAO lithography technique. Weng et al. demonstrated a fabrication method based on the local oxidation nanopatterning of graphene using AFM. ${ }^{12} 25 \mathrm{~nm}$-wide GNRs were fabricated by local anodic oxidation (LAO) lithography which has the advantage of monitoring the device parameters (such 


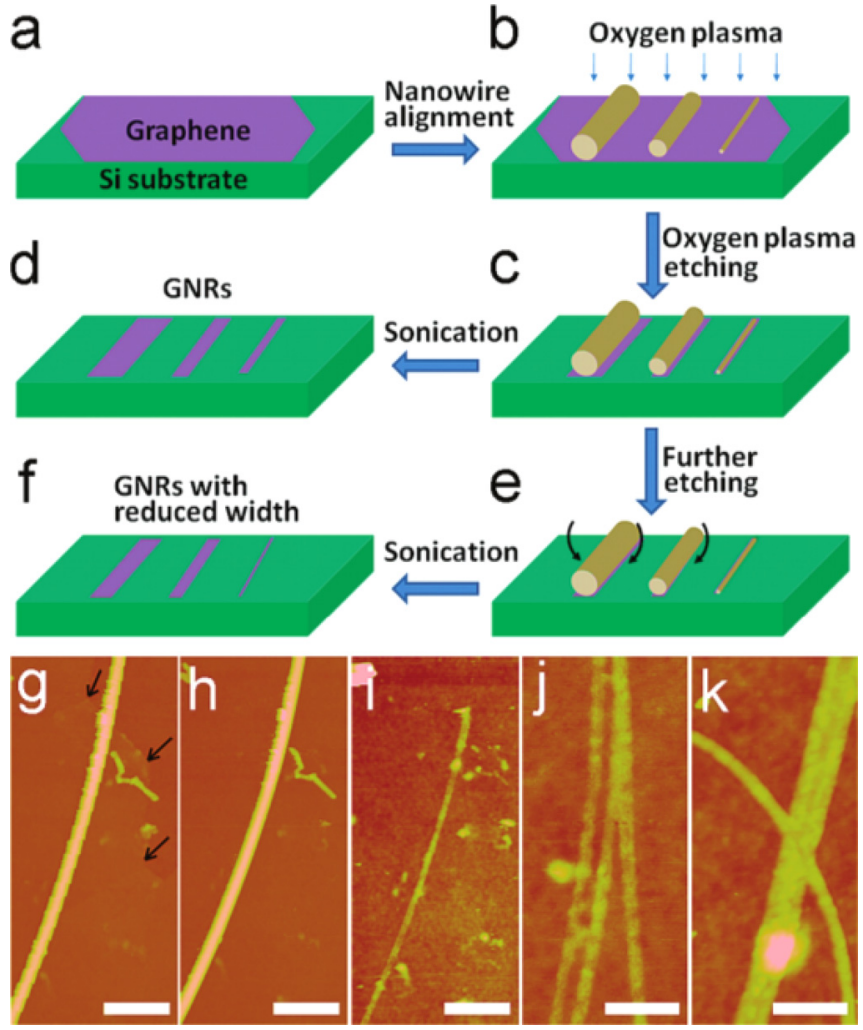

FIG. 3. (a)-(f) The schematic fabrication process of obtaining GNRs by oxygen plasma etch with a nanowire etch mask and $(\mathrm{g})$ and $(\mathrm{h})$ are AFM images of a nanowire etch mask lying on top of a graphene flake before $(\mathrm{g})$ and after (h) the oxygen plasma etch. The scale bars are $300 \mathrm{~nm}$ and $100 \mathrm{~nm}$ in ((g)-(i)) and $((\mathrm{j})$ and $(\mathrm{k}))$, respectively. Reprinted with permission from Bai et al., Nano Lett. 9, 2083 (2009). Copyright 2009 American Chemical Society.

as dimensions or electrical conduction) during the fabrication process. The same technique was also used by Masubuchi et al. in single-layer, bilayer, and multilayer graphenes, and $55 \mathrm{~nm}$-wide GNRs were fabricated. ${ }^{13}$

Unzipping is a technique that cuts multiwalled carbon nanotube (MWCNT) crossway to form GNRs having width which depends on the diameter of the CNT. Kosynkin et al. fabricated GNRs by longitudinally unzipping MWCNT using the oxidation technique. ${ }^{14}$ Such a lengthwise cutting was demonstrated for the first time. The same methodology was also used by Jiao et al. to produce smooth edge and narrow width GNRs. ${ }^{15}$ In their study, plasma etching of nanotubes in a polymer film was reported and GNRs were obtained by unzipping MWCNTs (see Fig. 3). The advantage of their approach is that GNRs can be realized with controlled widths and edge structures with high quality by controlling the placement and alignment of CNTs. It was also pointed out that the use of fewwalled CNTs leads to the production of sub-10 nm GNRs with bandgaps which are sufficient for room-temperature transistor applications. Jiao et al. reported the synthesis of pristine fewlayer NRs by unzipping gas-phase oxidized MWCNTs using mechanical sonication in an organic solvent. ${ }^{16}$ It was reported that the fabricated GNRs are of very high quality with very smooth edges. In addition, the produced GNRs have the highest electrical conductance and mobility ever reported to date (up to $5 e^{2} / \mathrm{h}$ and $1.500 \mathrm{~cm}^{2} / \mathrm{V}$ s for ribbons $10-20 \mathrm{~nm}$ in width). It was also shown that the GNRs have minimal defects and little edge roughness which is seen through their phase-coherent transport and their Fabry-Perot interference properties (Fig. 4).

As mentioned before, fabricating GNRs with wellordered edges and controllable widths is an important issue for their applications in nanoscale devices. Sprinkle et al. reported the synthesis of self-organized GNRs on a templated SiC substrate which was prepared by using scalable photolithography and microelectronics processing. ${ }^{17}$ It was pointed out that damaging the edges of GNRs by violent cutting processes such as $\mathrm{O}_{2}$ etching is eliminated by the contraction of top-down and bottom-up lithographies. Their results also demonstrated that the growth of graphene on non-traditional crystal faces is useful in device fabrication and for producing GNRs on a large scale.

The synthesis of large scale GNRs with high precision directly on insulating or semiconducting substrates is difficult. Jacobberger et al. reported the synthesis of GNRs on the $\mathrm{Ge}(001)$ substrate via chemical vapor deposition (CVD) technique. ${ }^{18}$ They observed that the growth of GNRs is selfaligning $3^{\circ}$ from the $\mathrm{Ge}(110)$ directions, is self-defining, and

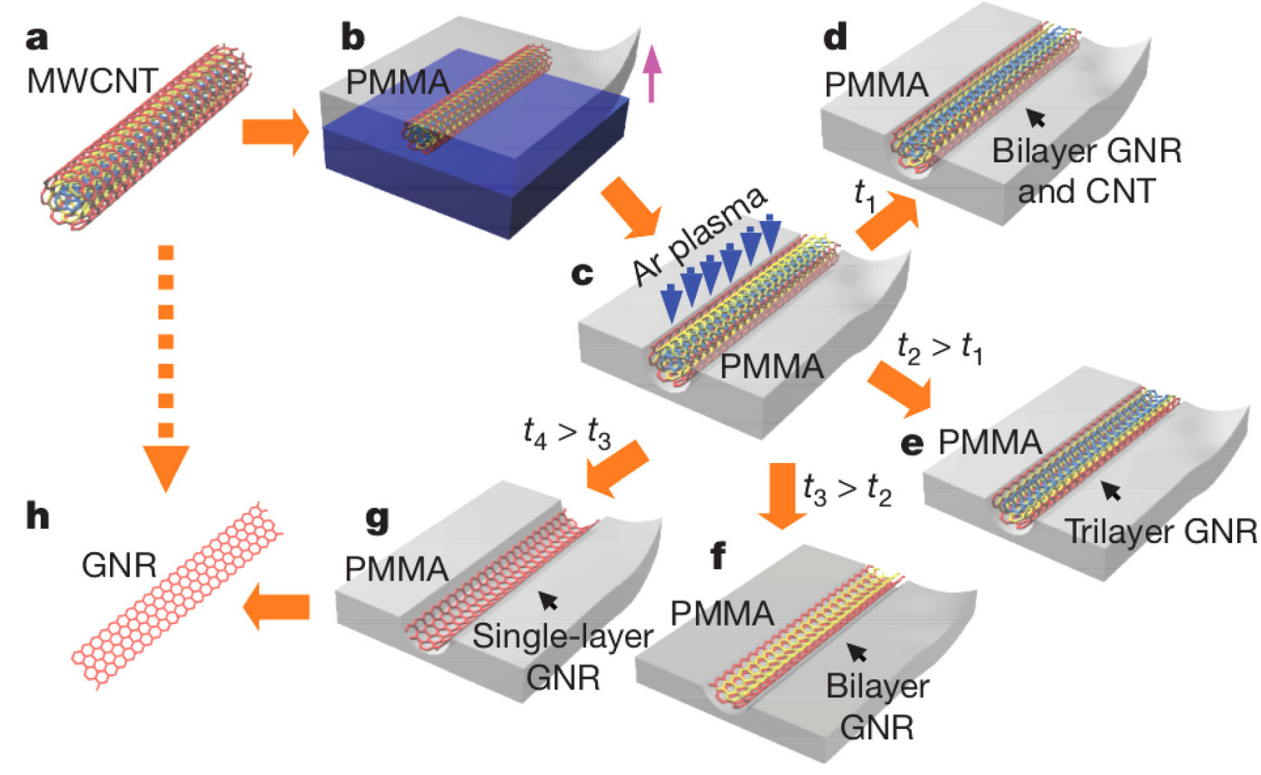

FIG. 4. The procedure of making GNRs from CNTs. (a) A pristine MWCNT, (b) the MWCNT deposited on a Si substrate, and (c) the coated PMMAMWCNT film then peeled from the Si substrate and exposed to Ar plasma. (d)-(g) Several possible products of GNRs for different times. The PMMA was removed at the end. Reprinted with permission from Jiao et al., Nature 458， 877 (2009). Copyright 2009 Nature Publishing Group. 
a
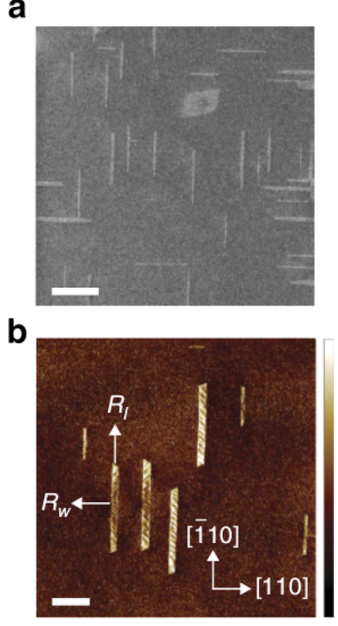

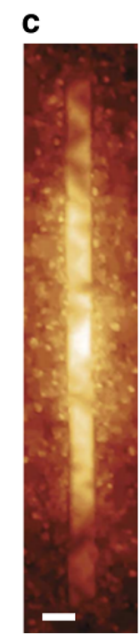

d

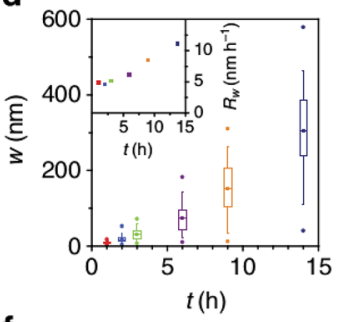

f $50 \quad$ g

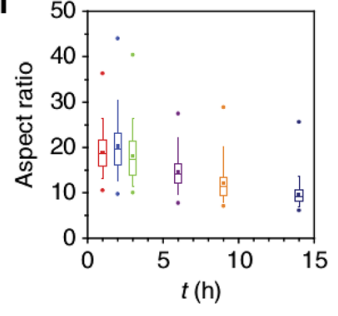

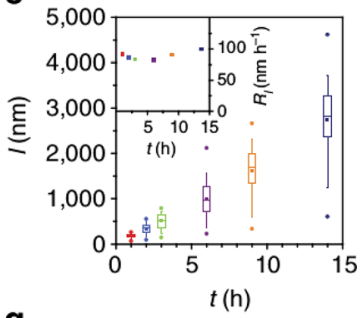

g

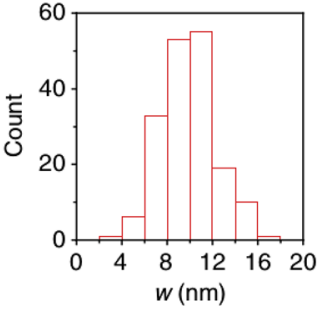

FIG. 5. Growth evolution of GNRs on Ge(001). (a) SEM, (b) AFM, and (c) STM (applied bias $-2 \mathrm{~V}$ and current $200 \mathrm{pA}$ ) images. In (a) and (b), the scale bar is $400 \mathrm{~nm}$ and $10 \mathrm{~nm}$ in (c). (d) Ribbon width, w, (e) I, and (f) aspect ratio, $\mathrm{f}$, plotted against time, $\mathrm{t}$. Insets of (d) and (e) are the mean $\mathrm{R} w$ and $\mathrm{R} l$, respectively. (g) Histogram of $\mathrm{w}$ from the $1 \mathrm{~h}$ growth in (d)-(f). Reprinted with permission from Jacobberger et al., Nat. Commun. 6, 8006 (2015). Copyright 2015 Nature Publishing Group.

has smooth armchair edges, tunable widths to $<10 \mathrm{~nm}$, and an aspect ratio $<70$ (see Fig. 5). It was mentioned that it is critical to operate in a regime in which the growth rate along the width direction is slow, $<5 \mathrm{~nm} / \mathrm{h}$, in order to realize highly anisotropic ribbons on semiconducting substrates. This is an important point for the integration of GNRs into future-hybrid integrated circuits.

In addition to the synthesis of GNRs, constructing different building blocks using synthesized NRs plays a crucial role in obtaining the effect of substrate. In the study performed by Han et al., self-assembly fabricated GNRs were connected end to end. ${ }^{19}$ Surface-assisted molecular assembly (SAMA) was used to synthesize and connect GNRs end to end both chemically and electronically. It was also mentioned that the $\mathrm{Cu}(111)$ substrate plays an important role for the molecules to form and connect the GNRs up to $50 \mathrm{~nm}$ long. The technique used in the study is also important for the understanding of the effect of substrate on the control of the alignment of GNRs. By using on-surface chemical reactions with an employed organoboron precursor, they realized boron-doped graphene NRs (BGNRs) having widths of $\mathrm{N}=7,14$, and 21. Additionally, as shown by Stampfer et al., two distinct voltage scales can be extracted experimentally which characterize parameter region of repressed conductance at low charge density in the ribbon. ${ }^{20}$

\section{B. Theoretical studies on graphene NRs}

For the investigation of fundamental properties of GNRs, theoretical studies using analytical approaches and computational tools are of importance. By using analytical approaches such as the tight-binding method, one may be able to calculate the magnetic and electronic properties of GNRs for a large number of atoms contained in the unit cell of the structure. In contrast, computational tools based on density functional theory (DFT) can handle only relatively narrow systems with a small number of atoms in the computational unit cell. However, DFT can produce accurate results with relatively small basis sets. Another advantage of more DFT-based computational tools is that it is very efficient for the ground state calculations of fundamental structural, magnetic, electronic, and optical properties. In addition, for overcoming the limitations of DFT, many functionals and approximations can be included such as the linear scaling approach for larger systems,

lattice dynamics, and cluster expansion for investigating finitetemperature effects, and also time-dependent DFT for electronic excitations.

As mentioned before, depending on the direction of the ribbon axis, GNRs can have zigzag or armchair edges which show very different physical properties. The magnetic interaction between the edge states in a GNR was first predicted by Lee $e t a l .^{21}$ It was found that the magnetism of edge states in GNRs can be well understood by considering the magnetic tails' interaction. At each side of the GNR, ferromagnetic (FM) interaction enhances the magnetic energy, while for the inter-edge interaction the antiferromagnetic coupling between the edge states is energetically favorable (see Fig. 6).

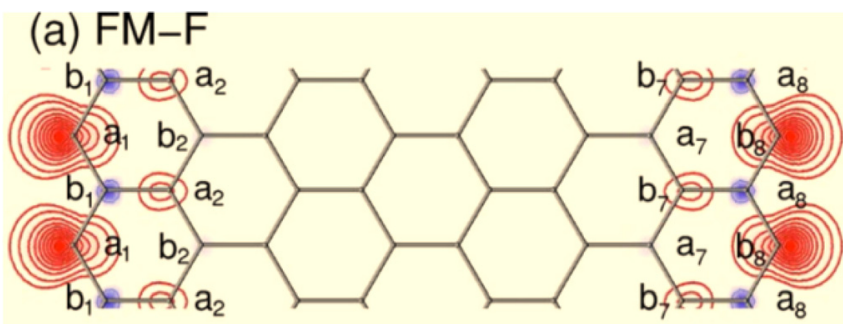

(b) FM-A

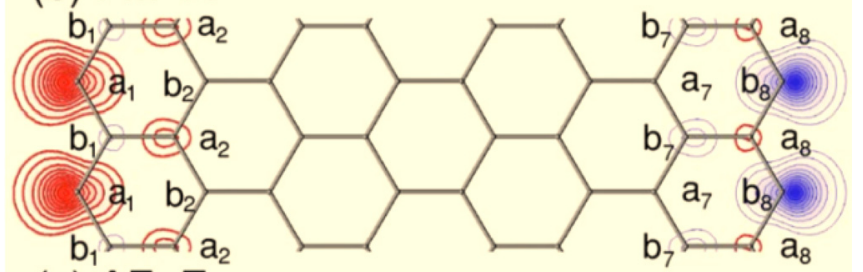

(c) $\mathrm{AF}-\mathrm{E}$

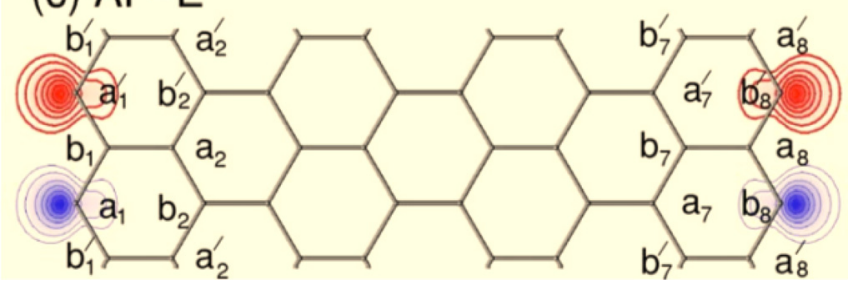

FIG. 6. Spin density contour plots for an isolated ribbon. (a), (b), and (c) are densities for FM-F, FM-A, and AF-E, respectively. Thicker lines indicate the up-spin density and thinner lines show the down-spin density. The contour plots are drown within the plane $0.6 \AA$ above the strip layer containing the center of carbon atoms. Reprinted with permission from Lee et al., Phys. Rev. B 72, 174431 (2005). Copyright 2005 American Physical Society. 
Modification of electronic properties by external effects such as edge-functionalization or applying external electric field is necessary for the materials which are not suitable for electronic applications. Son et al. predicted the half-metallicity in GNRs by using first-principles calculations. ${ }^{22}$ They showed that half-metallicity is realizable under applied in-plane homogeneous electric field across the zigzag edges in GNRs. They found that the ground state of ZGNR has a bandgap which is inversely proportional to the ribbon width. In addition, they calculated an energy splitting of $\sim 0.52 \mathrm{eV}$ which is independent of the width of the ZGNR if $\mathrm{N} \geq 8$. However, under applied electric field, one of the spin orientations closes its bandgap, while the other one widens it as seen Fig. 7. The study points out that the half-metallic behavior of ZGNRs can be controlled by an applied external transverse electric field. In addition, the same behavior, half-metallic character in ZGNRs, was also investigated by Kan et $a l^{23}$ The modification of the electronic properties of ZGNRs under functionalization of its edges by functional groups: $\mathrm{H}, \mathrm{NH}_{2}, \mathrm{NO}_{2}$, and $\mathrm{CH}_{3}$ was also investigated. It was reported that the spin-down gap becomes narrower when the ribbon width increases. Another point of the study is that some of the wide ribbons functionalized with $\mathrm{NO}_{2}$ groups at one edge and $\mathrm{CH}_{3}$ groups at the other edge have halfmetallic character. Moreover, the half-metallic ribbons can be more stable than the $\mathrm{H}$-saturated ones if the concentration of large functional groups is reduced. Son $e t$ al. investigated the energy bandgaps in GNRs as a function of their widths. ${ }^{24}$ It was found that the origin of the bandgap in AGNRs is due to both by quantum confinement and by the crucial effect of the particular type of edges. Edge magnetization was found in ZGNRs. Those first principles calculations predict that the bandgap of AGNRs is separated into 3 families $\left(\Delta_{3 p+1}>\Delta_{3 p}\right.$ $>\Delta_{3 p+2}$, where $p$ is a positive integer) as a function of the ribbon width and there are no metallic NRs (Table I).

In order to realize experimental bandgap of materials, it is important to include screened Coulomb interaction through the theoretical tools. Yang et al. investigated the quasiparticle energies and bandgap of GNRs by using a first-principles many-electron Green's function approach within the GW approximation. ${ }^{25}$ The calculated quasiparticle bandgaps exhibit significant self-energy corrections of $0.5-3.0 \mathrm{eV}$ for ribbons of width $2.4-0.4 \mathrm{~nm}$ for both AGNRs and ZGNRs. However, the calculated quasiparticle bandgaps are in the range of $1-3 \mathrm{eV}$ for GNRs of width $2-1 \mathrm{~nm}$ which is promising for applications in nanoelectronics. Plasmon excitations in metallic AGNRs were studied by Shylau et al. and exact analytical expression for the polarization function of Dirac fermions was obtained. ${ }^{26}$ At finite temperatures, the full polarization function exhibits temperature and position of the chemical potential independent behavior. Cervantes-Sodi et al. studied the effect of edge-functionalization and substitutional doping on the electronic and magnetic properties of GNRs. ${ }^{27}$ It was reported that in ZGNRs the spin degeneracy can be broken by chemical modification. In addition, edge functionalization of AGNRs does not significantly affect the bandgap. Moreover, single-edge functionalization of ZGNRs leads to half-semiconductors with different bandgaps for each spin state and a spin-polarized half-semiconductor or a semiconductor-metal transition can be observed which are important for spin-filtering device applications. It was mentioned that high concentrations of oxygen $(\mathrm{O})$ functionalization of the ribbon edges produce metallic-character ZGNRs, while for low concentration of O ZGNRs exhibit half-semiconducting behavior. In another study, Wassmann et al. investigated the stability, the geometry, and the electronic property of GNRs edges saturated with $\mathrm{H}$ atoms. ${ }^{28}$ AFM ZGNRs were found to be stable only at very low ultravacuum pressures. However, for more standard conditions, mono- and di-hydrogenated structures are the most stable ones for AGNRs, while di- and two monohydrogenated ZGNRs are the most stable structures. It was

a

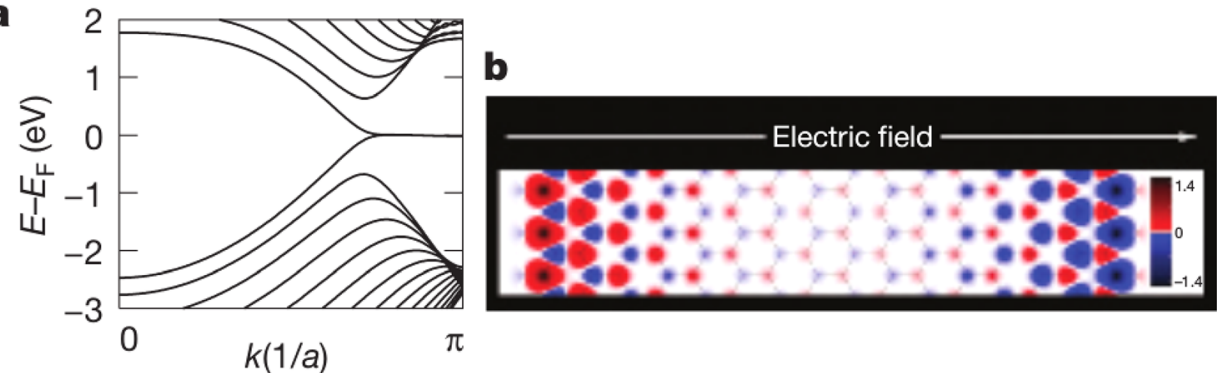

C

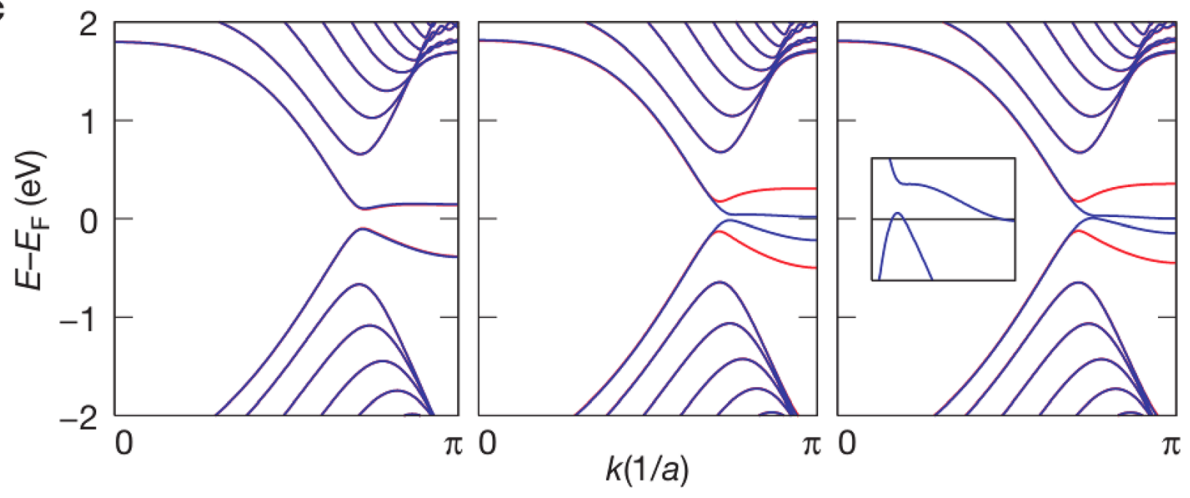

FIG. 7. (a) The spin-unpolarized band structure of 16-ZGNR, (b) the spatial distribution of the charge difference between two spin states for the ground state under zero-electric field (the scale bar is in units of $10^{-2} \mathrm{e} / \AA^{2}$ ), and (c) the spin-resolved band structures of a 16ZGNR under applied 0.0, 0.05, and $0.1 \mathrm{~V} / \AA$ electric field from left to right. The red and blue lines denote two different spin-states, respectively. Reprinted with permission from Son et al., Nature 444, 347 (2006). Copyright 2006 Nature Publishing Group. 
TABLE I. Synthesis technique, general electronic behavior, width dependence of electronic bandgap and magnetic ground state of recently demonstrated NRs.

\begin{tabular}{|c|c|c|c|c|c|}
\hline Material & References & Synthesis technique & $\begin{array}{c}\text { Electronic } \\
\text { behavior }(\mathrm{eV})\end{array}$ & $\begin{array}{l}\text { Width-bandgap } \\
\text { dependence }\end{array}$ & $\begin{array}{l}\text { Magnetic } \\
\text { ground state }\end{array}$ \\
\hline Graphene (zigzag) & $\begin{array}{c}5 \text { and } 7 \\
9 \text { and } 10 \\
11 \\
12 \\
14 \text { and } 15\end{array}$ & $\begin{array}{l}\text { Bottom-up approach } \\
\text { Rational approach } \\
\text { Anisotropic etching } \\
\text { LAO lithography } \\
\text { Unzipping MWCNTs }\end{array}$ & $\mathrm{SC}(1-3 \mathrm{eV})$ & & FM-AFM \\
\hline Graphene (armchair) & $\begin{array}{c}5,7 \text {, and } 8 \\
10 \text { and } 16 \\
6 \\
11 \\
12 \text { and } 13 \\
14\end{array}$ & $\begin{array}{l}\text { Bottom-up approach } \\
\text { Rational approach } \\
\text { SACMP } \\
\text { Anisotropic etching } \\
\text { LAO lithography } \\
\text { Unzipping MWCNTs }\end{array}$ & $\mathrm{SC}(0.05-1.3 \mathrm{eV})$ & $1 / \mathrm{w}$ & NM \\
\hline Graphane & 37 & $\ldots$ & $\mathrm{SC}$ & $\sim\left(3.42+\exp ^{-N}\right)$ & NM \\
\hline $\mathrm{MoS}_{2}$ (zigzag) & $\begin{array}{l}40 \\
42\end{array}$ & $\begin{array}{l}\text { Electrochemical/chemical } \\
\text { encapsulated in CNTs }\end{array}$ & M & w-independent & FM \\
\hline $\mathrm{MoS}_{2}$ (armchair) & 46 & $\ldots$ & $\mathrm{SC}(0.51-0.56 \mathrm{eV})$ & Weakly dependent & NM \\
\hline $\mathrm{WS}_{2}$ & $\begin{array}{l}55 \\
56\end{array}$ & $\begin{array}{c}\text { Encapsulated in CNTs } \\
\text { unzipping of } \mathrm{WS}_{2} \text { nanotubes }\end{array}$ & $\begin{array}{c}\text { SC (armchair) } \\
(0.37-0.52 \mathrm{eV}) \mathrm{M} \text { (zigzag) }\end{array}$ & Weakly dependent & $\begin{array}{l}\text { NM (armchair), } \\
\text { FM (zigzag) }\end{array}$ \\
\hline $\operatorname{ReS}_{2}$ & 64 & CVD & $\mathrm{SC}(0.92 \mathrm{eV})$ & Very weak dependence & NM \\
\hline $\mathrm{TiSe}_{2}$ & 67 & $\ldots$ & $\mathrm{SC}(0.005-0.790 \mathrm{eV})$ & $\sim \exp ^{-N}$ & NM \\
\hline $\mathrm{GaS}$ & 70 and 71 & $\ldots$ & $\begin{array}{c}\text { SC (armchair) } \\
(1.45 \mathrm{eV}) \mathrm{HM} \text { (zigzag) }\end{array}$ & Oscillates with w & $\begin{array}{l}\text { NM (armchair) } \\
\text { FM (zigzag) }\end{array}$ \\
\hline $\mathrm{ZnSe}$ & $\begin{array}{l}74 \\
75\end{array}$ & $\begin{array}{l}\text { Laser ablation of pressed-powders } \\
\text { solvothermal procedure }\end{array}$ & $\mathrm{SC}(2.60 \mathrm{eV})$ & w-independent & $\ldots$ \\
\hline SnSe & 77 & $\ldots$ & $\begin{array}{c}\text { SC (armchair) } \\
(0.92 \mathrm{eV}), \mathrm{M} \text { (zigzag) }\end{array}$ & Inversely proportional & NM \\
\hline $\mathrm{TiS}_{3}$ & 79 & Mechanical exfoliation & $\mathrm{SC}(1.0 \mathrm{eV})$ & w-independent & FM \\
\hline $\mathrm{ZrS}_{3}$ & 87 and 88 & CVT & $\mathrm{SC}$ & $\ldots$ & \\
\hline Phosphorene & 89 & Top-down method & $\begin{array}{c}\mathrm{SC} \text { (armchair) } \\
(0.5-0.8 \mathrm{eV}), \mathrm{M} \text { (zigzag) }\end{array}$ & Inversely proportional & NM \\
\hline Silicene & $\begin{array}{c}\text { 100, 101, and } 104 \\
103\end{array}$ & $\begin{array}{l}\text { Deposition of Si atoms } \\
\text { under UHV }\end{array}$ & $\begin{array}{l}\text { M or SC (armchair) } \\
\text { SC (zigzag) }\end{array}$ & $\begin{array}{l}\text { Family-behavior (armchair) } \\
\text { Inversely proportional (zigzag) }\end{array}$ & $\begin{array}{l}\text { NM (armchair) } \\
\text { AFM (zigzag) }\end{array}$ \\
\hline Germanene & 116 & $\cdots$ & $\begin{array}{c}\mathrm{SC} \text { (armchair) }(0-0.9 \mathrm{eV}) \\
\mathrm{SC} \text { (zigzag) }(0.08-0.16 \mathrm{eV})\end{array}$ & $\begin{array}{l}\sim \exp ^{-N} \\
\sim \exp ^{-N}\end{array}$ & $\begin{array}{l}\text { NM (armchair) } \\
\text { AFM (zigzag) }\end{array}$ \\
\hline Stanene & 120 & $\cdots$ & $\begin{array}{l}\text { SC (armchair) }(0.05-0.35 \mathrm{eV}) \\
\mathrm{SC} \text { (zigzag) }(0.075-0.16 \mathrm{eV})\end{array}$ & $\begin{array}{l}\text { Oscillatory-behavior } \\
\text { Inversely proportional }\end{array}$ & $\begin{array}{l}\text { NM (armchair) } \\
\text { AFM (zigzag) }\end{array}$ \\
\hline h-BN & 125 and 126 & Unwrapping MWBNNTs & SC (armchair), M or SC (zigzag) & & $\begin{array}{l}\text { NM (armchair), } \\
\text { NM or FM (zigzag) }\end{array}$ \\
\hline h-AlN & 134 & $\cdots$ & $\begin{array}{c}\mathrm{SC} \text { (armchair) }(0.53-3.23 \mathrm{eV}) \\
\mathrm{SC}, \mathrm{SM} \text {, or M (zigzag) }(0-2.98 \mathrm{eV})\end{array}$ & $\begin{array}{l}\text { Monotonic-decreasing } \\
\text { Monotonic-decreasing }\end{array}$ & $\begin{array}{c}\mathrm{NM} \\
\mathrm{M}\end{array}$ \\
\hline
\end{tabular}

mentioned in the study that the knowledge of the structure and stability of the edges of GNRs is critical for the control of experimental conditions of GNR formation.

Determining conductance and energy scale for quantum transport properties of materials is important for their applications in atomic-scale devices. Biel et al. studied the quantum transport properties of chemically doped GNRs having width smaller than $4 \mathrm{~nm} .{ }^{29}$ It was found that $\mathrm{B}$ and $\mathrm{N}$ impurities in GNRs yield resonant backscattering. The features of this resonant backscattering strongly depend on the position of the dopants, the symmetry of the structure, and the width of the GNRs. In addition, they observed an unusual acceptor-donor transition in ZGNRs. In the study of Scuracchio et al., the role of atomic vacancies and boundary conditions on the ballistic thermal transport properties of GNRs were investigated. ${ }^{30}$ It was found that edge and central localized single atomic vacancies do not affect the low-energy transmission function of in-plane phonon modes. However, they reduce considerably the contributions of the flexural modes. Similarly, Zhang et al. investigated the resonant tunneling properties of S- and U-shaped GNRs and showed that the tunneling current is tunable under varying the Fermi energy. ${ }^{31}$ In addition, tunability of resonant tunneling was realized by the change of width of the leads and by the lack of external gates. Petrovic and Peeters reported the existing of Fano resonances in narrow GNRs of different widths and edge types. ${ }^{32}$ In another study, Droscher et al. investigated the conductance of GNRs and found that single or multiple quantum dots dominate the conductance depending on the ribbon length, density of electrons, and temperature. ${ }^{33}$ Huang et al. constructed a field effect transistor consisting of a metalsemiconductor-metal junction by using substitutionally doped ZGNRs. ${ }^{34}$ It was shown that a metal-semiconductor transition can be seen in ZGNRs when edges are doped with $\mathrm{B}$ or $\mathrm{N}$ atoms. It was also reported that the bandgap of the doped ZGNR depends strongly on the NR width and showed a linear dependency with doping concentration. The simulated field 
(a)

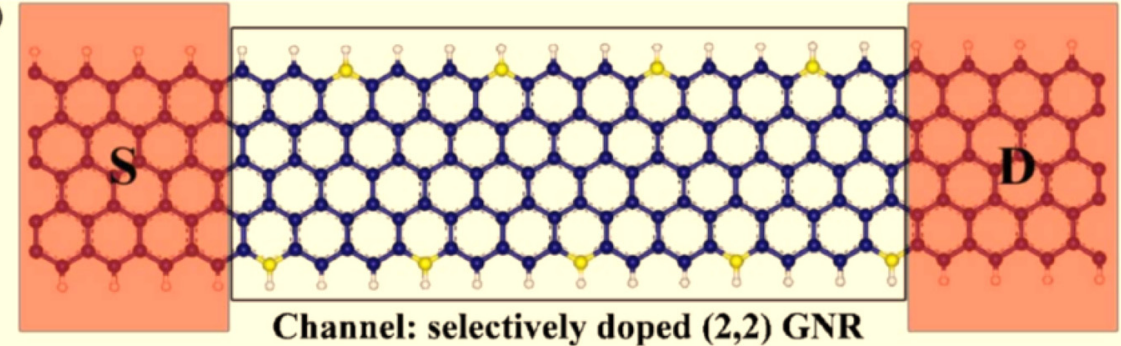

(b)
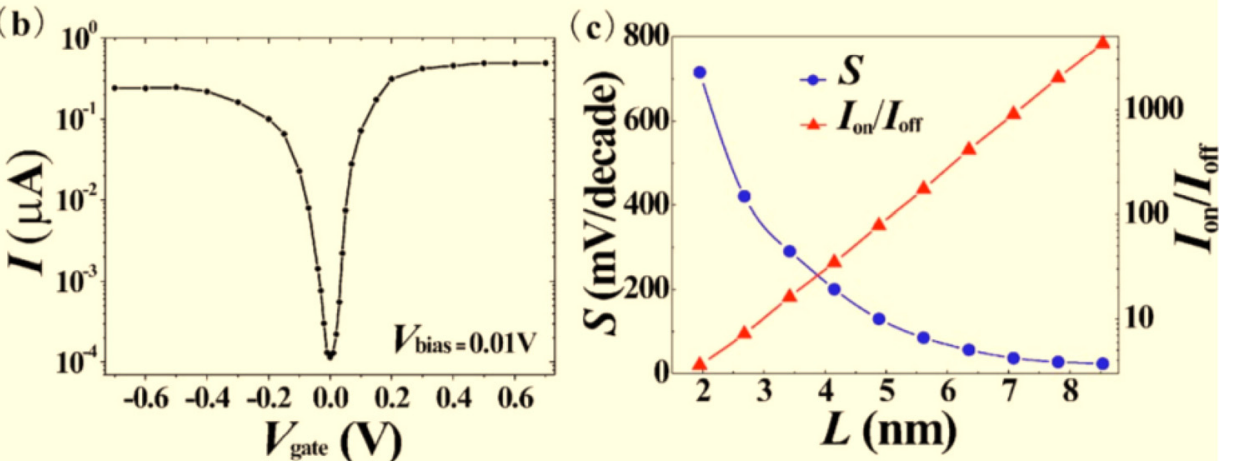

FIG. 8. (a) The schematic structure of the FET constructed from a single $(2,2)$ ZGNR. The semiconducting region is obtained by doping the edges with $\mathrm{N}$ atoms (the center region). (b) Simulated $\mathrm{I}-\mathrm{V}_{\text {gate }}$ curves for $\mathrm{N}$-doped ZGNR-FETs under applied bias voltage of $0.01 \mathrm{~V}$, the length of the channel is $8.54 \mathrm{~nm}$, and the linear doping concentration is $0.13651 / \AA$. Reprinted with permission from Appl. Phys. Lett. 91, 253122 (2007). Copyright 2007 AIP Publishing LLC. effect transistor (FET) was found to exhibit excellent performance characteristics (see Fig. 8). Ren et al. studied electronic and elastic transport properties of AGNR-based junctions. ${ }^{35}$ Their results of the I-V characteristics of various junctions demonstrate negative differential resistance (NDR) phenomena.

Full functionalization is another effective way to modify the characteristic properties of graphene-based materials. As demonstrated by Elias et al., surfaces of graphene can be chemically hydrogenated. ${ }^{36}$ The resulting structure, graphene, is a honeycomb structure of $\mathrm{sp}^{3}$ linked hydrocarbons. Sahin et al. investigated electronic and magnetic properties of graphene NRs and found that H-passivated zigzag and armchair graphene NRs are NM semiconductors, while the bare zigzag GNRs were found to have AFM ordering at the edges. ${ }^{37}$ It was shown that the bandgaps of both zigzag and armchair graphene NRs depend exponentially on the width and it decreases as the width increases.

\section{NRS OF TRANSITION METAL DICHALCOGENIDES}

Tremendous interest on graphene has also led to the emergence of novel two dimensional materials such as transition metal dichalcogenides (TMDs). The first synthesized member of TMDs, notably $\mathrm{MoS}_{2},{ }^{38}$ has aroused strong interest in the field of 2D materials due to its robust chemical properties, suitable bandgap for optoelectronic applications, ${ }^{39}$ and high on/off ratio of FET at room-temperature. ${ }^{38}$ Different from graphene, as mentioned, 2D monolayer $\mathrm{MoS}_{2}$ has a suitable bandgap which makes this material an important candidate for optoelectronic applications. Therefore, in the 1D NR form, the gap is tunable by its width and therefore this structure can be expected to be a promising material for technological applications.

\section{A. NRs of $\mathrm{MoS}_{2}$}

\section{Experimental studies on $\mathrm{MoS}_{2}$}

Advances in experimental techniques also opened the possibility of the synthesis of nanoscale-wide $\mathrm{MoS}_{2}$ NRs in various ways. For their synthesis, especially electron irradiation and CNT-based encapsulation techniques have been widely used.

The first successful synthesis of thin $\mathrm{MoS}_{2}$ NRs was realized by performing a two-step electrochemical/chemical synthetic method, reported by $\mathrm{Li}$ et al ${ }^{40}$ The synthesized $\mathrm{MoS}_{2}$ NRs were produced above $800^{\circ} \mathrm{C}$ (see Fig. 9) and they are $50-800 \mathrm{~nm}$ in width and $3-100 \mathrm{~nm}$ thick. The optical absorption measurements revealed that these $\mathrm{MoS}_{2}$ NRs are direct gap semiconductors with a bandgap of $1.95 \mathrm{eV}$. It can be understood that the $\mathrm{MoS}_{2}$ NRs with widths in the range of $50-800 \mathrm{~nm}$ correspond to the same electronic

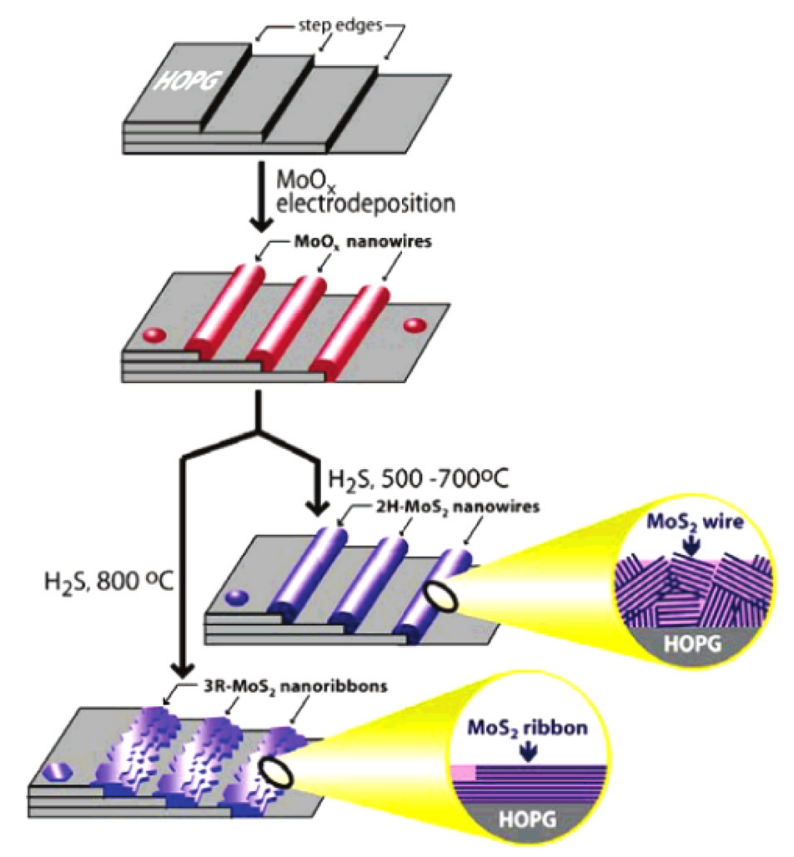

FIG. 9. The schematic representation of the electrochemical/chemical method for synthesizing polycrystalline $\mathrm{MoS}_{2}$ nanowires and NRs on graphite surfaces. Reprinted with permission from Li et al., J. Phys. Chem. B 109, 3169 (2005). Copyright 2005 American Chemical Society. 
bandgap which indicates that the bandgap is saturated to a certain value and the effect of the width of the NRs will be very small.

Electron irradiation process which uses high-energy electrons is another way of fabricating different morphologies of materials. Liu et al. reported the successful fabrication of $\mathrm{MoS}_{2} \mathrm{NRs}$ with uniform width of $0.35 \mathrm{~nm}$ that were formed under electron irradiation ${ }^{41}$ (see Fig. 10). It was found that the fabricated NR is a semiconductor with a bandgap of $0.77 \mathrm{eV}$ and it has a Young modulus of $300 \mathrm{GPa}$. Moreover, fabricated $\mathrm{MoS}_{2}$ NR can sustain 9\% tensile strain before fracture. It was also pointed out that although it is difficult to control geometries and properties of NRs below $10 \mathrm{~nm}$-width, the technique used in the study is important for such purposes.

Integrating mixed low-dimensional nanomaterials is a fascinating and challenging issue for controlling the dimensions of the fabricated material. Wang et al. synthesized mixed and stable low-dimensional nanomaterial in which $\mathrm{MoS}_{2}$ NRs are encapsulated in CNTs that they called nanoburritos. ${ }^{42}$ The width of the encapsulated $\mathrm{MoS}_{2} \mathrm{NRs}$ is uniform and is in the range of $1-4 \mathrm{~nm}$, while the number of layer is down to 1-3 layers. The edge of the synthesized NRs was identified to be zigzag shape by using HRTEM which was supported by DFT calculations. It was pointed out that the synthesis technique used in the study can be extended to the synthesis of other ultranarrow TMD NRs. Transistor made of $\mathrm{MoS}_{2} \mathrm{NR}$ was fabricated and its physical properties were investigated by Liu et al. ${ }^{43}$ The channel conductance in the fabricated FET was found to scale linearly with the channel width which is a signature that there is no evidence of edge damage. It was found that transistors having thin channel thickness have a larger threshold voltage $\left(V_{T}\right)$. This shift is associated with the width scaling. In addition, there are
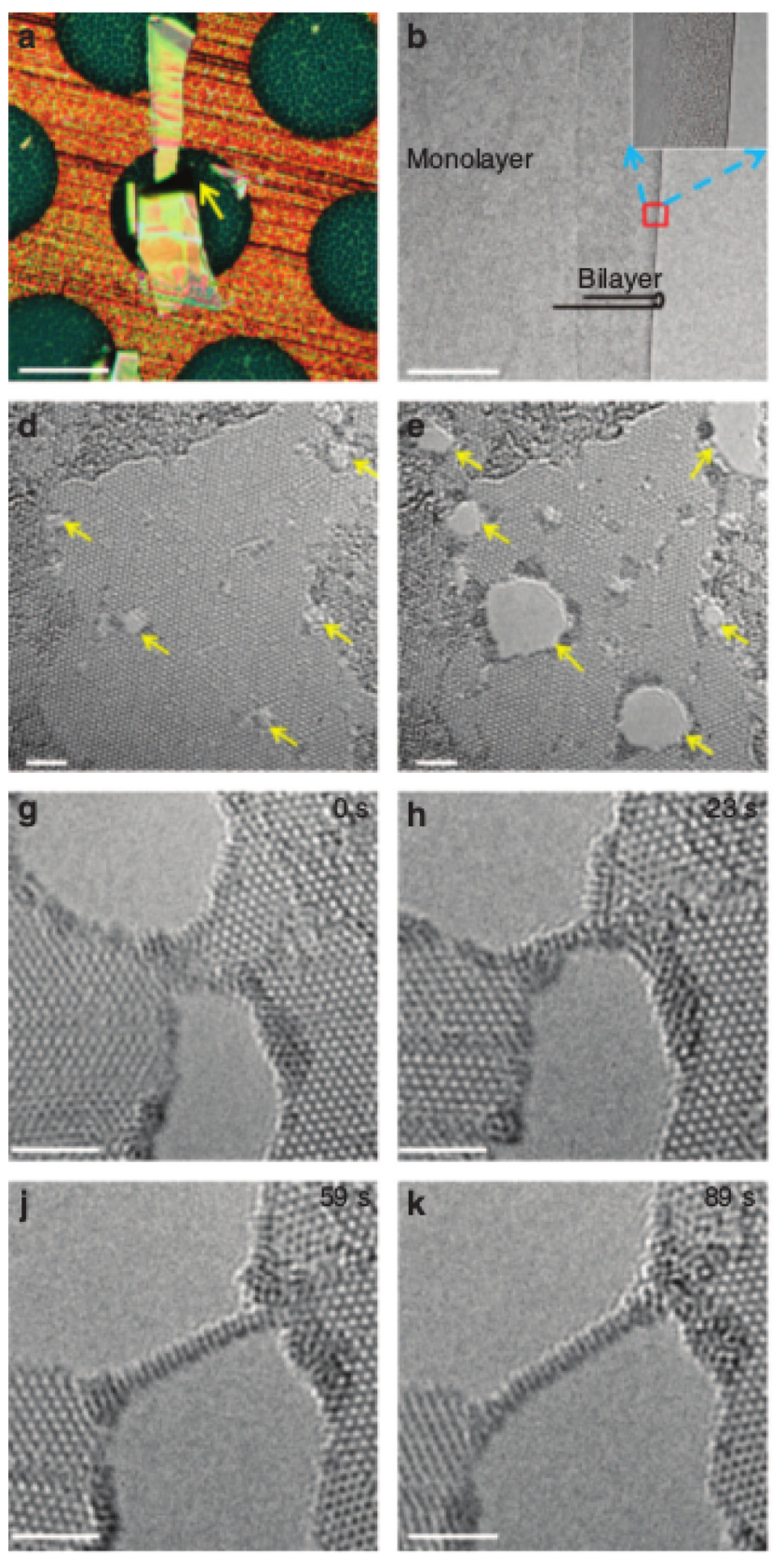
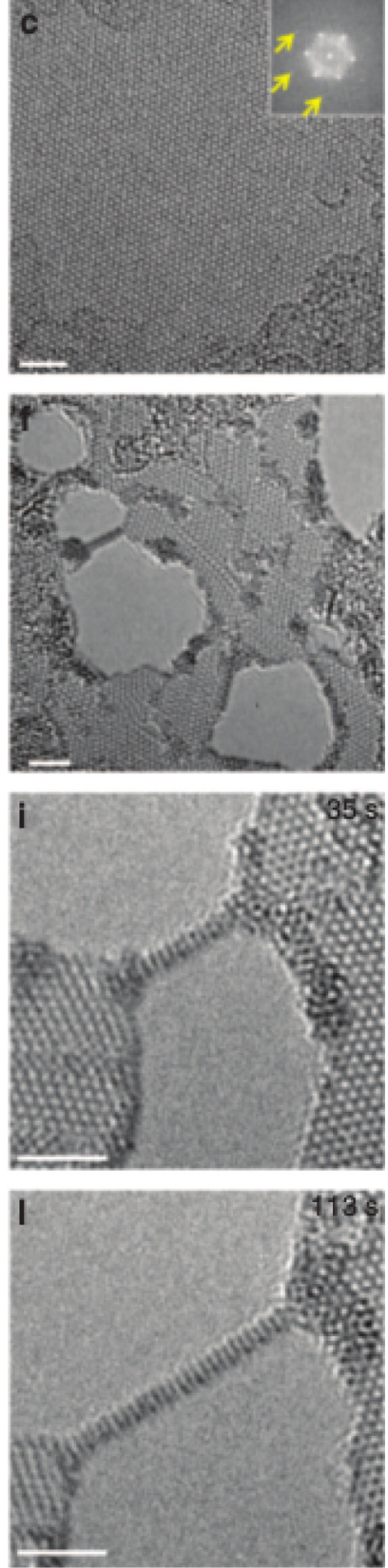

FIG. 10. (a) Few-layer $\mathrm{MoS}_{2}$ flake on a TEM grid (scale bar is $100 \mu \mathrm{m}$ ). (b) TEM images of the thinnest region given in part (a). The straight edge reveals the single layer form of $\mathrm{MoS}_{2}$ flake (scale bar is $100 \mathrm{~nm}$ ). (c) HRTEM data obtained on the unfolded regions. (d) The initial $\mathrm{MoS}_{2}$ including small irradiation-induced vacancies. (e-I) Time series of the formation of a suspended ribbon under electron irradiation (scale bar is $2 \mathrm{~nm}$ in c-I). Reprinted with permission from Liu et al., Nat. Commun. 4, 1776 (2013). Copyright 2013 Nature Publishing Group. 


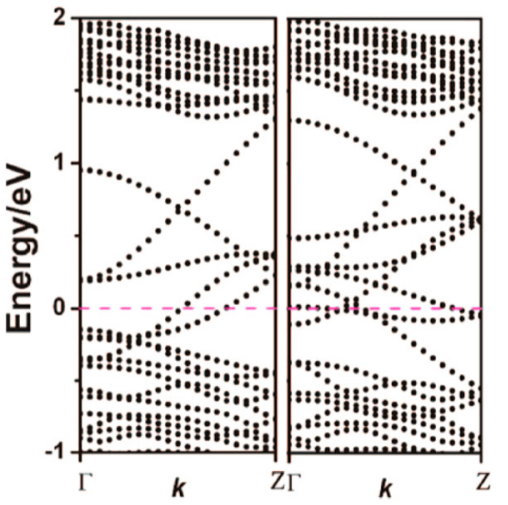

(a)

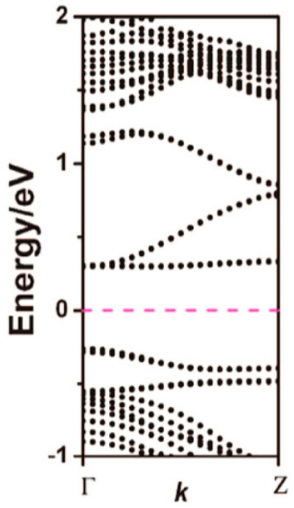

(b)

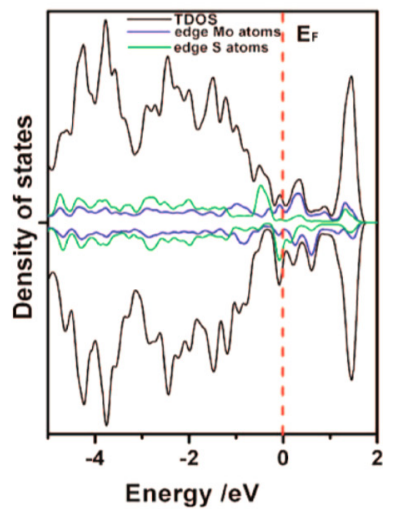

(c)
FIG. 11. (a) The spin-polarized band structure for $8-\mathrm{ZMoS}_{2} \mathrm{NR}$ (left one is for spin up and right figure is for spin down states, respectively). (b) The spin-polarized band structure for 15$\mathrm{AMoS}_{2}$ NR. (c) Total density of states (TDOS) and local density of states (LDOS) for $8-\mathrm{ZMoS}_{2} \mathrm{NR}$ (top, spin up; bottom, spin down). Reprinted with permission from Li et al., J. Am. Chem. Soc. 130, 16739 (2008). Copyright 2008 American Chemical Society. also studies on the edge site activity of $\mathrm{MoS}_{2}$ for hydrogen evolution reaction (HER). ${ }^{4,45}$ In those studies, it was mainly mentioned that the number of $\mathrm{S}$ edge sites plays an active role in the HER and by controlling the size of these $S$ edge sites it is possible to engineer the HER activity in $\mathrm{MoS}_{2}$.

\section{Theoretical studies on $\mathrm{MoS}_{2}$}

Although there are only a few experimental studies on $\mathrm{MoS}_{2}$ NRs, a much larger number of theoretical studies are available which predict the physical properties of $\mathrm{MoS}_{2}$ NRs. Li et al. investigated the high stability and unusual electronic and magnetic properties of $\mathrm{MoS}_{2} \mathrm{NRs}$ with either zigzag $\left(\mathrm{ZMoS}_{2}\right)$ or armchair $\left(\mathrm{AMoS}_{2}\right)$ edges. ${ }^{46}$ It was found that NRs with zigzag edges show FM and metallic behavior independent of the ribbon width and thickness as shown in Fig. 11. However, the NRs with armchair edges are NM semiconductors and the bandgap depends on the width of the ribbon and saturates to a value of $0.56 \mathrm{eV}$ after a certain width of $\mathrm{MoS}_{2}$ NR. It was also pointed out that the $\mathrm{ZMoS}_{2}$
NRs are more stable than $\mathrm{AMoS}_{2}$ ones and both are more stable than the reported $\mathrm{MoS}_{2}$ nanoclusters. Ataca et al. studied the mechanical and electronic properties of $\mathrm{MoS}_{2} \mathrm{NRs}$ and also their defected structures. ${ }^{47}$ It was predicted that the $\mathrm{MoS}_{2}$ NRs are stiff quasi one-dimensional structures with a high in-plane stiffness. The calculated phonon frequencies for armchair $\mathrm{MoS}_{2}$ NRs exhibit four acoustic branches as with real frequencies which indicate their dynamical stability. In addition, magnetic moments and spin-polarization of $\mathrm{ZMoS}_{2}$ can be reduced by edge passivation with $\mathrm{H}$ atoms. Furthermore, $\mathrm{NM} \mathrm{AMoS}_{2}$ can possess a net magnetic moment depending on the adsorption site of foreign atoms and the type of vacancy defect created in the structure. The creation of $\mathrm{MoS}_{2}$ triple vacancy in $\mathrm{AMoS}_{2}$ results in a significant net magnetic moment of $2 \mu_{B}$ which is promising for magnetic applications of $\mathrm{MoS}_{2}$ (Fig. 12).

Tuning the physical properties of materials by external fields is an important issue for using those materials in various applications. Applying external electric field and strain are two important ways to achieve such tuning. Dolui et al.
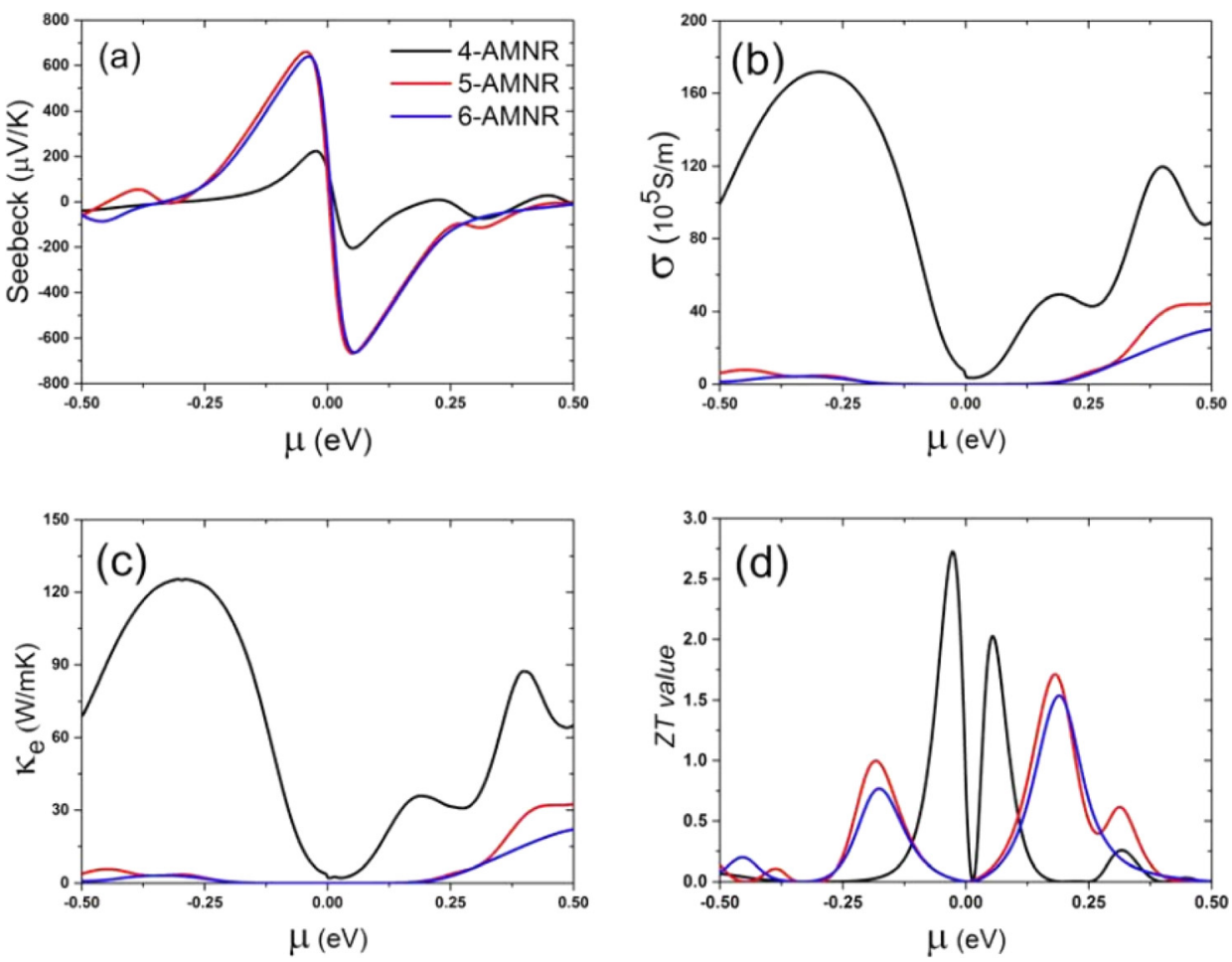

FIG. 12. The calculated electronic transport coefficients and ZT values of $\mathrm{N}-\mathrm{AMoS}_{2} \mathrm{NRs}(\mathrm{N}$ indicates the width of the NR) as a function of chemical potential at $300 \mathrm{~K}$ : (a) Seebeck coefficient, (b) electrical conductivity, (c) electronic thermal conductivity, and (d) ZT value. Reprinted with permission from Appl. Phys. Lett. 105, 133113 (2014). Copyright 2014 AIP Publishing LLC. 
investigated the effect of an electric field on the physical properties of $\mathrm{AMoS}_{2} \mathrm{NRs}^{48}$ An applied external transverse electric field was found to significantly reduce the bandgap of $\mathrm{AMoS}_{2} \mathrm{NR}$ and results in a metal-insulator transition beyond a certain critical field. In addition, in the case of bilayer, $\mathrm{AMoS}_{2}$ NRs an indirect to direct gap transition is induced by the applied field. The critical electric field decreases with increasing ribbon width. Ouyang et al. studied the effect of hydrogenation of $\mathrm{AMoS}_{2}$ NRs in terms of edge magnetization and reported that bare and fully passivated $\mathrm{AMoS}_{2}$ NRs are NM semiconductors but FM edge states can be induced by patterned hydrogenation. ${ }^{49}$ In addition, in the presence of an applied transverse electric field, partial edge hydrogenation can change a small size $\mathrm{AMoS}_{2}$ NR from semiconductor to metal or semimetal. Thus, hydrogenation is an important way to tune the magnetic and electronic properties of $\mathrm{AMoS}_{2} \mathrm{NRs}$.

The possible battery capacity of $\mathrm{MoS}_{2}$ NRs was investigated for different types of atoms. Yang et al. studied the $\mathrm{ZMoS}_{2}$ NRs as a promising cathode material for rechargeable $\mathrm{Mg}$ batteries by first-principles calculations. ${ }^{50} \mathrm{By}$ calculating the possible adsorption sites and diffusion kinetics for $\mathrm{Mg}$ atom on $\mathrm{ZMoS}_{2} \mathrm{NR}$, they found that the top of the Mo site at the edge of the NR is the most favorable site for $\mathrm{Mg}$ atom. In addition, a maximum theoretical capacity of 223.2 $\mathrm{mA} \mathrm{h} / \mathrm{g}$ was predicted for double site $\mathrm{Mg}$ adsorption case. The activation barrier for a $\mathrm{Mg}$ atom to diffuse between the two Mo-top sites was calculated to be only $0.48 \mathrm{eV}$. It was pointed out in this study that in terms of its $\mathrm{Mg}$ storage capacity and diffusion kinetics, $\mathrm{ZMoS}_{2}$ NRs can be a good candidate for rechargeable $\mathrm{Mg}$ batteries. $\mathrm{Li}$ et al. investigated the adsorption and diffusion of $\mathrm{Li}$ atoms on $\mathrm{ZMoS}_{2}$ NRs for possible applications in Li-ion batteries. ${ }^{51}$ It was reported that going from $\mathrm{MoS}_{2}$ nanosheets to $\mathrm{ZMoS}_{2} \mathrm{NRs}$, the binding energy and the mobility of $\mathrm{Li}$ atoms increase which makes the $\mathrm{ZMoS}_{2} \mathrm{NR}$ a promising cathode materials for Li-ion batteries with high power densities and fast charge/discharge rates.

The thermoelectric properties of $\mathrm{AMoS}_{2}$ NRs were investigated by Fan et al. ${ }^{52} \mathrm{AMoS}_{2}$ NRs could be optimized to exhibit very good thermoelectric performance. The figure of merit (ZT) value of the considered NRs was found to be width dependent and can take values as high as 2.7 at room temperature. It was pointed out that if the width or edge of the synthesized NR can be controlled, then $\mathrm{AMoS}_{2} \mathrm{NRs}$ could become very promising thermoelectric materials. Zhang et al. investigated the phonon thermal conductivity in $\mathrm{MoS}_{2}$ NRs and found that in contrast to GNRs, the thermal conductivity of $\mathrm{MoS}_{2}$ NRs is insensitive to the width, the length, and the type of NR edge. ${ }^{53}$ In addition, Liu et al. studied the anisotropic thermal conductivity of $\mathrm{MoS}_{2} \mathrm{NRs}$ in a more general manner. ${ }^{54}$ Since the previous thermal transport studies were limited to zigzag and armchair directions only, they investigated anisotropic thermal transport properties of $\mathrm{MoS}_{2}$ NRs in different directions and reported that thermal conductivity has a local maximum at the direction of $19.1^{\circ}$. Furthermore, calculated full-band phonon dispersion revealed that over $90 \%$ of the thermal conductivity is contributed by the LA mode in $\mathrm{MoS}_{2}$ NRs.

\section{B. NRs of $\mathrm{WS}_{2}$}

Tungsten disulfide $\left(\mathrm{WS}_{2}\right)$, a member of the TMD family, possesses a layered structure in its bulk form which is quite similar to $\mathrm{MoS}_{2}$. WS 2 mainly exhibits the properties of dry lubricants and it was used in conjunction with other materials as catalyst for hydrotreating of crude oil. In addition, it can also be used in high temperature and high pressure applications. Similar to $\mathrm{MoS}_{2}$, when thinned down to its monolayer form, an indirect-to-direct optical transition was observed. The monolayer form of $\mathrm{WS}_{2}$ was also successfully isolated.

The 1D NR form of $\mathrm{WS}_{2}$ was first synthesized by Wang et al. as encapsulated in CNTs. ${ }^{55}$ Single and double walls CNTs were used as templates and successfully fabricated $\mathrm{WS}_{2}$ NRs were realized with smooth edges. The reported widths of the $\mathrm{WS}_{2}$ NRs vary between 1 and $2 \mathrm{~nm}$ and the number of layers is $1-3$ depending on the diameter of the CNTs. It was found that in contrast to bulk $\mathrm{WS}_{2}$ which has a NM semiconducting ground state, whereas free standing ultra-narrow zigzag $\mathrm{WS}_{2}$ NRs can have magnetic or NM metallic ground state depending on the edge passivation. However, the $\mathrm{WS}_{2}$ NRs with armchair edges always remain NM semiconductors having a narrow gap. In another study, Nethravathi et al. synthesized $\mathrm{WS}_{2}$ NRs by chemically unzipping $\mathrm{WS}_{2}$ nanotubes using $\mathrm{Li}$ atoms. ${ }^{56} \mathrm{WS}_{2}$ nanotubes were intercalated by $\mathrm{Li}$ atoms and the nanotubes reacted with various solvents and broke into NR pieces. The synthesized NRs are about 10-12 layers and have a width of about $100 \mathrm{~nm}$ which is wider than that synthesized by Wang et al. ${ }^{55}$

There exist several theoretical studies by investigating the physical properties of these NRs which we will summarize now in this part of the review. Zhang et al. investigated the structural, electronic, and magnetic properties of $\mathrm{WS}_{2}$ NRs and found that the properties of the NRs are strongly affected by the type of the edges. ${ }^{57}$ Armchair-edged $\mathrm{WS}_{2}$ NRs are NM semiconductors, while the zigzag-edged ones are magnetic metallic structures. In addition, it was shown that the electronic and magnetic properties of $\mathrm{WS}_{2}$ NRs are, respectively, sensitive to external electric field and applied strain. Ouyang et al. studied the effect of edge hydrogenation on the physical properties of $\mathrm{WS}_{2}$ NRs. ${ }^{58}$ It was found that zigzag $\mathrm{WS}_{2}$ NRs conserve its FM semiconducting behavior upon edge hydrogenation, while $\mathrm{WS}_{2}$ NRs with armchair edges convert from NM to magnetic ground state when at least one edge is partially hydrogenated. Furthermore, it was found that depending on the width of $\mathrm{WS}_{2} \mathrm{NRs}$, magnetic moment of zigzag $\mathrm{WS}_{2}$ NRs displays periodic behavior.

In addition to their fundamental physical properties, the chemical activity of $\mathrm{WS}_{2}$ NRs was theoretically investigated by considering their interactions with certain molecules. Kvashnin et al. ${ }^{59}$ studied the interaction of 1-octanethiol molecule with $\mathrm{Li}$ ions to simulate the experiment performed by Nethravathi et al. It was found that unzipping $\mathrm{WS}_{2}$ nanotubes occurs due to the intercalation of lithiated multi wall nanotubes by 1-octanethiol molecules. Since the lithiated nanotubes expand, unzipping is possible with 1-octanethiol molecule. It was shown that the penetration of this molecule 
between walls of the nanotube stretches the nanotube up to an amount of $8.9 \%$ which enables the unzipping of the nanotube to obtain $\mathrm{WS}_{2} \mathrm{NRs}$. In another theoretical study, LopezUrias et al. investigated the electronic, magnetic, optical, and edge reactivity properties of $\mathrm{WS}_{2}$ NRs. ${ }^{60}$ They showed that zigzag-edged $\mathrm{WS}_{2} \mathrm{NRs}$ exhibit a FM metallic ground state with magnetic moments localized at the $\mathrm{W}$ and $\mathrm{S}$ edge atoms. The NRs with armchair edges display semiconducting behavior. It was also reported that there is a strong optical polarization anisotropy which enhances a well defined absorption intensity peak having a polarization along the axis of the NR. The reactivity of the edges of $\mathrm{WS}_{2} \mathrm{NRs}$ was tested by making the edges interact with molecules. Results demonstrated that water molecule at the armchair edge dissociates into $\mathrm{OH}$ attached to a $\mathrm{W}$ atom and the $\mathrm{H}$ which is attached to the $\mathrm{S}$ atom on the edge.

\section{In-plane-anisotropic NRs: $\operatorname{ReX}_{2} \mathrm{~s}$}

In a single crystal, the physical properties often differ with the crystallographic orientation and these materials are known as anisotropic materials. Monolayer crystals of these in-plane anisotropic materials are another subclass of dichalcogenides. Due to their orientation dependent electronic, magnetic, optical, and thermal properties, there are many application areas for these anisotropic materials. From the family of TMDs, monolayer $\operatorname{ReS}_{2}$ has been recently synthesized and gained a lot of interest owing to its distorted and anisotropic structure. ${ }^{61}$ It was reported that this new monolayer material behaves as its bulk form both electronically and vibrationally. It was shown that when $\mathrm{ReS}_{2}$ is thinned from bulk to monolayer, it still remains as a direct bandgap semiconductor and its Raman spectrum shows no dependence on the number of layers. $\mathrm{ReSe}_{2}$, another member of TMDs family which has also a distorted crystal structure like $\mathrm{ReS}_{2}$, has been successfully synthesized in its monolayer form. ${ }^{62}$ It was found that the fabricated FET with monolayer $\mathrm{ReSe}_{2}$ exhibits p-type conductivity at room temperature with a higher mobility than that of the few-layered one. In addition, it was reported that the number of layers strongly affects the band-gap and mobility in $\mathrm{ReSe}_{2}$.

Although its 2D monolayer form was synthesized and its physical properties were analyzed, 1D NR form of $\mathrm{ReSe}_{2}$ has not been synthesized yet. However for quasi-1D structure of $\mathrm{ReSe}_{2}$, the response of optical, electrical, and magnetic properties to external stain was investigated by Yang et $a l .{ }^{63}$ Nanoscale local strain can modulate the optical bandgap, induce magnetism, and change the electronic and vibrational response of $\mathrm{ReSe}_{2}$ before the fracture point.

The NR form of $\mathrm{ReS}_{2}$ was successfully synthesized by He et al. ${ }^{64}$ The direct growth of high-quality $\mathrm{ReS}_{2}$ layers and NRs by the CVD method was reported. It was found that by tuning the growing time for $\mathrm{ReS}_{2}$ layers, $\mathrm{ReS}_{2}$ NRs can be synthesized (see Fig. 13). It was pointed out that controlling the growth time for $\mathrm{ReS}_{2}$ different morphologies of the material can be synthesized.

In addition to this experimental study, there are also theoretical investigations on the electronic properties of $\mathrm{ReS}_{2}$ NRs. Yu et al. investigated the effect of ribbon width on the

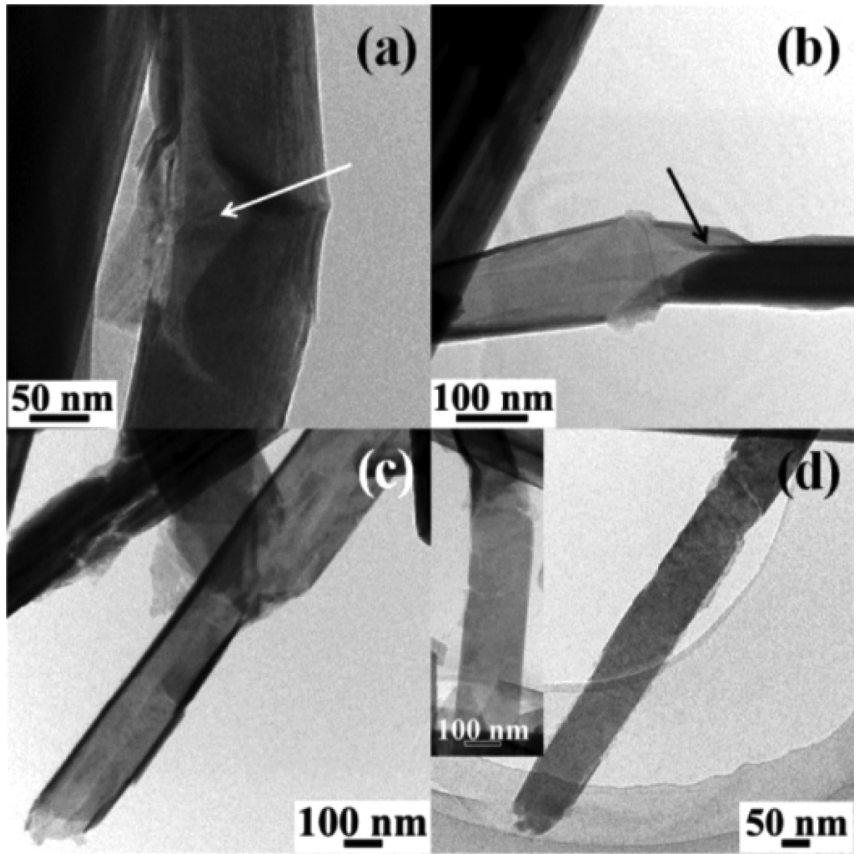

FIG. 13. (a)-(d) Bright-field TEM images of $\mathrm{WS}_{2}$ NRs which are produced by unzipping of lithiated $\mathrm{WS}_{2}$ NTs with the help of octanethiol. (a) and (b) demonstrate the incubation stages for the unzipping process. Reprinted with permission from Nethravathi et al., ACS Nano 7, 7311 (2013). Copyright 2013 American Chemical Society.

electronic properties of $\mathrm{ReS}_{2}$ NRs. ${ }^{65}$ They showed that the direct bandgap of $\mathrm{ReS}_{2} \mathrm{NRs}$ depends very weakly on the width of the NR and after a certain width the bandgap saturates to a value of $0.92 \mathrm{eV}$. It was pointed out that when compared with other TMDs members, the reduction in the bandgap of monolayer $\mathrm{ReS}_{2}$ when thinned to NR form is smaller (Fig. 14).

\section{Octahedrally coordinated NRs: $\mathrm{TiSe}_{2}$}

As mentioned before, TMDs can possess $1 \mathrm{H}$ or $1 \mathrm{~T}$ symmetry in their ground state depending on the constituent atoms. As a member of 1T-TMDs family, $\mathrm{TiSe}_{2}$ is an intrinsically layered material in its bulk form which possesses both superconducting and charge density wave (CDW) ordering and thus, it has been widely studied due to these remarkable physical properties.

The monolayer form of this material was first synthesized by Peng et al. by molecular beam epitaxy (MBE) growth on $\mathrm{SiC}(0001)$ substrate. ${ }^{66}$ Upon growing monolayer $\mathrm{TiSe}_{2}$ crystals, two different types of defects were observed: interstitial and Se vacancy. It was shown that the concentration of these defects depends critically on the substrate temperature. In addition, it was reported that the band structure of monolayer $\mathrm{TiSe}_{2}$ differs fundamentally from its bulk counterpart.

The NR structure of $\mathrm{TiSe}_{2}$ was proposed theoretically; however, it has not been synthesized yet. Ozaydin et al. investigated the electronic and magnetic properties of $1 \mathrm{~T}$ phase of $\mathrm{TiSe}_{2}$ NRs (1T-TiSe 2 NRs) which is a member of TMDs family. ${ }^{67}$ The electronic bandgap of the $1 \mathrm{~T}-\mathrm{TiSe}_{2}$ NRs decreases exponentially as the width of the ribbon gets wider over $20 \AA$. In addition, for ultranarrow zigzag edge 

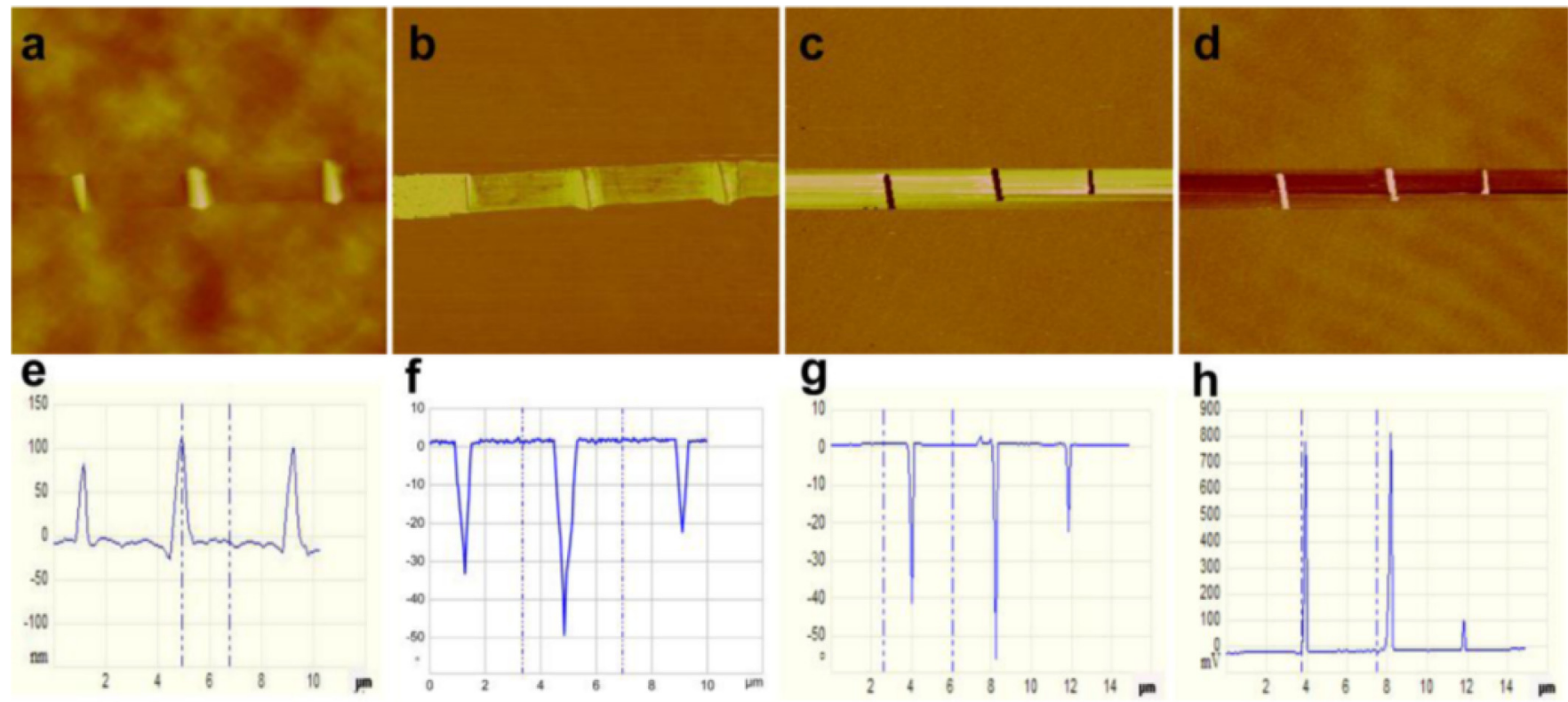

FIG. 14. Magnetic force microscopy (MFM) measurements of quasi-1D ReSe 2 . (a) AFM topography, (b) phase, (c) MFM phase, and (d) MFM amplitude for wrinkled flakes on gel-film substrate. (e)-(h) The profiles for images in (a)-(d). Reprinted with permission from Yang et al., Nano Lett. 15, 1660 (2015). Copyright 2015 American Chemical Society.

1T-TiSe 2 NRs, odd-even oscillations were shown. Calculations on the magnetic properties demonstrated that all $1 \mathrm{~T}-\mathrm{TiSe}_{2} \mathrm{NRs}$, even zigzag or armchair edged, have nonmagnetic ground states.

\section{MONOCHALCOGENIDE NRS}

In addition to TMDs, compounds having monochalcogenide atom in their chemical formula have been the focus of recent interest due to their potential applications in various fields. These materials can be classified into different sub-classes depending on the non-chalcogenide atom in the structure. For instance, crystals with $\mathrm{Ge}, \mathrm{Sn}$, or $\mathrm{Pb}$ atoms correspond to group IV mono-chalcogenide family, while structures with $\mathrm{Ga}$ or In atoms stand for post-transition metal mono-chalcogenide (PTMC) family. Some of these crystals have layered bulk structures and like many other layered materials, when dimensional reduction is achieved they possess different physical properties.

\section{A. Gallium monochalcogenides: GaS, GaSe}

Gallium sulfide $(\mathrm{GaS})$ and gallium selenide $(\mathrm{GaSe})$ are two members of PTMC and have a layered crystal structure in their bulk forms. The 2D monolayer forms of these PTMC structures were successfully synthesized and gained a lot of interest due to their unique physical properties. Meng et al. presented a functionalization technique to control the optical properties of both CVD grown and exfoliated Ga-based PTMCs using pyridine $\left(\mathrm{C}_{5} \mathrm{H}_{5} \mathrm{~N}\right)$ organic intercalant molecules. ${ }^{68}$ It was found that the molecules intercalate between GaSe layers and cause an increase in PL intensity of the crystal. In another study, Cai et al. built a type-I Ga-chalcogenide heterojunction by transferring exfoliated few-layer GaSe onto bulk gallium telluride (GaTe) sheets. ${ }^{69}$ It was reported that GaSe layers provide photo-excited electrons and holes to the GaTe sheets transferring from outer layers to the interface. It was pointed out that the optical performance of the heterostructure can be tuned by combining different thicknesses of the Ga-chalcogenide layers.

Although its nanowire, nanotube, and nanobelt forms were synthesized, NRs of GaS crystal have not been synthesized, so far. However, there are theoretical studies predicting some unique properties of the NR form of GaS. Wang et al. investigated the electronic properties and edge effects of GaS NRs and found that the electronic properties of the GaS NRs are strongly affected by the edge structure. ${ }^{70}$ Zigzag-edged GaS NRs are metallic with spin-polarized edges independent of the $\mathrm{H}$ passivation, while the armchairedged GaS NRs display indirect-gap semiconducting behavior. It was shown that for armchair NRs the bandgap oscillates as the width of the NR increases and saturates to a constant value for wide NRs. Additionally, Zhou et al. investigated the intrinsic half-metallic character with FM coupling in GaS NRs. ${ }^{71}$ They demonstrated that this magnetic property occurs at the Ga-dominated edge of GaS NRs due to $p$ states of $\mathrm{S}$ and $s$ and $p$ states of Ga atoms. Moreover, it was shown that this novel half-metallic behavior with FM coupling is rather robust especially for GaS NRs with large width and thickness. It is important to note that half-metallic behavior with FM coupling can be induced in GaS NRs without any external effects such as an external electric field or applying strain.

\section{B. Mono-selenide NRs: ZnSe and SnSe}

$\mathrm{ZnSe}$, another material containing chalcogenide atom, is a member of group II-VI semiconductors and its 1D structures, ZnSe NRs, were recently synthesized. ${ }^{72,73}$ Jiang et al. successfully produced ZnSe NRs using laser ablation of ZnSe pressed-powders. ${ }^{74}$ Their experiments on the characterization of $\mathrm{ZnSe}-\mathrm{NRs}$ revealed that it has a perfect 
wurtzite-2H single-crystal structure. In addition, microRaman scattering analysis showed that both the longitudinal optic (LO) and the transverse optic (TO) phonon peaks of the $\mathrm{ZnSe}$ NRs are shifted toward low frequencies relative to its bulk values due to small-size and the large-surface effects. It was also pointed out that this synthesis technique can be useful for the synthesis of other II-VI non-oxide semiconductor NRs. Later in another study, Zhao et al. fabricated vertically aligned $\mathrm{ZnSe-NR}$ arrays. ${ }^{75}$ The $\mathrm{ZnSe}-\mathrm{NRs}$ were grown on a $\mathrm{Zn}$ foil by using a simple solvothermal procedure. It was reported that the synthesized NRs are $100-300 \mathrm{~nm}$ in width and about $4 \mathrm{~nm}$ in thickness, while their lengths are about $2 \mu \mathrm{m}$. Their results demonstrated that the $\mathrm{ZnSe}-\mathrm{NRs}$ are good field emitters when compared with many other nanostructures with a low turn-on field of $5 \mathrm{~V} / \mu \mathrm{m}$.

$\mathrm{SnSe}$ is another crystal of chalcogenide based materials. Its $2 \mathrm{D}$ structure was recently synthesized as a new member of the transition metal chalcogenide (TMC) family. Li et al. successfully synthesized the single-layer single-crystalline $\mathrm{SnSe}$ nanosheet with thickness of about $1.0 \mathrm{~nm} .^{76}$ One-pot solution method was used to prepare single-crystalline $\mathrm{SnSe}$ and it was reported that 1,10-phenanthroline (Phen) plays an important role in determining the morphology of the $\mathrm{SnSe}$ because in the absence of Phen three-dimensional $\mathrm{SnSe}$ nanoflowers were obtained although all other parameters are the same.

The 1D form of this material was also considered theoretically (see Fig. 15). Huang et al. investigated the edge-, width-, and strain-dependent electronic properties of $\mathrm{SnSe}$ NRs and found that electronic properties can be tuned by: (i) altering the width parameter, (ii) applying tensile or compressive strain in zigzag SnSe NRs, and (iii) modification of edge type. ${ }^{77}$ In addition, they found that semiconductor-metal or metal-semiconductor transition can be realized by controlling all these parameters. Tyagi et al. studied the thermoelectric properties of SnSe NRs. ${ }^{78}$ It was found that armchair NRs with widths less than $47 \AA$ display semiconducting character, while the zigzag-edged NRs narrower than $52 \AA$ are metallic. For the armchair SnSe NRs with $6 \AA$ width, a relatively high

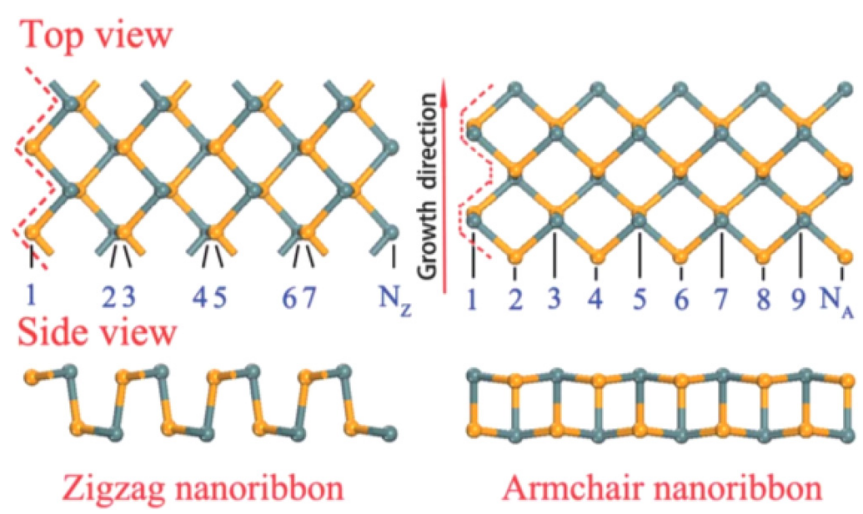

FIG. 15. Structures of SnSe NRs for zigzag (left panel) and armchair (right panel) and the definitions of the width parameters. Reprinted with permission from Huang et al., RSC Adv. 4, 6933 (2014). Copyright 2014 Royal Society of Chemistry.
Seebeck coefficient (SC) of $1720 \mu \mathrm{V} / \mathrm{K}$ and a low thermal conductivity were reported. It was pointed out that SnSe NRs may provide thermoelectric performance similar to that of monolayer and low-temperature bulk phases of SnSe.

\section{NRS OF TRI-CHALCOGENIDES}

Like the TMD family, transition metal trichalcogenides (TMTs) exhibit layered structures with weak interlayer van der Waals interactions in their bulk form. Bulk TMTs having the formula of $\mathrm{MX}_{3}(\mathrm{M}=\mathrm{Ti}, \mathrm{Zr}, \mathrm{Hf} ; \mathrm{X}=\mathrm{S}, \mathrm{Se}, \mathrm{Te})$ have been widely studied for several decades. However, only the 2D monolayer form of titanium three sulfide $\left(\mathrm{TiS}_{3}\right)$ was successfully isolated and reported to be a direct gap semiconductor. Before its successful isolation of 2D monolayer form, 1D NR of $\mathrm{TiS}_{3}$ was synthesized.

\section{A. High electron mobility NRs: $\mathrm{TiS}_{3}$}

One of the most recently announced NR structure is $\mathrm{TiS}_{3}$ that belongs to the trichalcogenides. Island et al. first isolated few-layer $\mathrm{TiS}_{3} \mathrm{NRs}$ that were used to fabricate FET. ${ }^{79,80}$ The fabricated devices show an ultrahigh photoresponse up to values of $2910 \mathrm{~A} / \mathrm{W}$. In addition, an n-type semiconducting behavior with mobilities of $2.6 \mathrm{~cm}^{2} / \mathrm{V} \mathrm{s}$ and on/ off ratio of $10^{4}$ was found for $\mathrm{TiS}_{3}$ NRs which make it an interesting material for photodetection and photovoltaic applications. NRs of $\mathrm{TiS}_{3}$ were also successfully synthesized by Barawi et al. ${ }^{81}$ The hydrogen storage capacity of $\mathrm{TiS}_{3}$ NRs was studied. The stability of $\mathrm{TiS}_{3}$ NRs was investigated by TGA (thermal gravimetric analysis) measurements which were carried out under argon and it was found that $\mathrm{TiS}_{3} \mathrm{NRs}$ are stable below $400{ }^{\circ} \mathrm{C}$. It was also found that the amount of the stored $\mathrm{H}$ increases as the $\mathrm{H}_{2}$ pressure increases up to 80 bars. In another study, Pawbake et al. carried out temperature-dependent Raman spectroscopy of $\mathrm{TiS}_{3} \mathrm{NRs}$ and compared it with that of $\mathrm{TiS}_{3}$ nanosheets. ${ }^{82}$ The Raman modes soften (red shift) as the temperature increases from 88 to $570 \mathrm{~K}$ which is related to the anharmonic vibrations of the lattice. This property can be an important mark in determining the temperature of $\mathrm{TiS}_{3}$ nanodevices by using Raman spectroscopy. It was also pointed out that the Raman modes of $\mathrm{TiS}_{3}$ NRs are less sensitive to temperature than that of $\mathrm{TiS}_{3}$ nanosheets which is related to lower interlayer coupling in the nanosheets. Another successful synthesis of $\mathrm{TiS}_{3} \mathrm{NRs}$ was achieved by Cui et al. and the time-resolved transient absorption measurements on $\mathrm{TiS}_{3}$ NRs were reported ${ }^{83}$ (see Fig. 16). It was found that time-resolved measurements reveal ultrafast injection, the thermalization, energy relaxation, and exciton formation of photocarriers occur on a subpicosecond time scale.

Although experimental studies on chalcogenide based NRs are limited, theoretical studies report the physical properties of these structures. Here, we review some of these important studies.

As mentioned above $\mathrm{TiS}_{3}$ was first successfully synthesized in NR form. After that, theoretical analyses of this structure were provided. Kang et al. studied the electronic properties, carrier mobility, and strain response of $\mathrm{TiS}_{3} \mathrm{NRs}$ and found that the edge type of the NR strongly affects the 

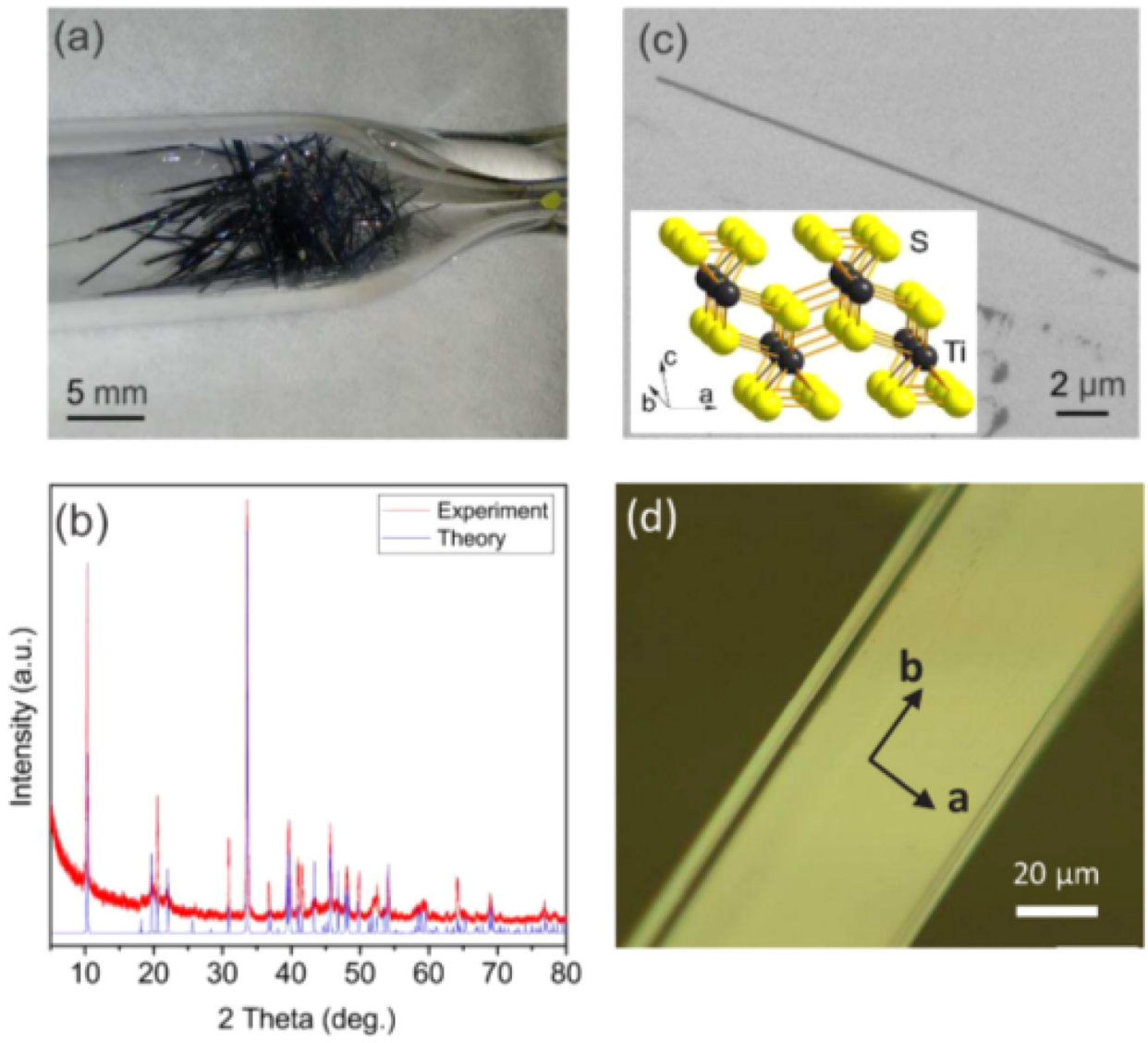

FIG. 16. Synthesis and characterization of $\mathrm{TiS}_{3}$ crystals. (a) An image of $\mathrm{TiS}_{3}$ single crystal formed on the walls of a quartz ampoule. (b) Corresponding powder X-ray diffraction pattern obtained both experimentally (red) and theoretically (blue). (c) SEM image of exfoliated $\mathrm{TiS}_{3}$ crystal on $\mathrm{Si} / \mathrm{SiO}_{2}$ substrate. The inset demonstrates the crystal structure of $\mathrm{TiS}_{3}$ with labelling crystallographic directions. (d) Optical microscopy image of a $\mathrm{TiS}_{3}$ NR. Reprinted with permission from Cui et al., ACS Appl. Mater. Interfaces 8, 18334 (2016). Copyright 2016 American Chemical Society. electronic properties. ${ }^{84}$ Depending on the edge type, $\mathrm{TiS}_{3}$ NRs can display metallic or semiconducting behavior which is independent of the ribbon width. This was reported as an important point because in an experiment there can exist NRs with different widths on the same substrate and all these ribbons will have the same electronic bandgap. In addition, it was reported that upon passivation of the edges of NRs by $\mathrm{H}$ atoms, the bandgap of semiconducting $\mathrm{TiS}_{3} \mathrm{NRs}$ is significantly increased. Furthermore, it was shown that the bandgap increases as the applied tensile strain is increased, demonstrating a direct-to-indirect bandgap transition.

\section{B. Quasi-1D behavior in $\mathrm{ZrS}_{3}$}

Zirconium trisulfide $\left(\mathrm{ZrS}_{3}\right)$, a member of the TMT family, is also a typical van der Waals (vdW) stacked layered material in its bulk form and can be isolated to its 2D monolayer form as many other layered materials. Although its 2D monolayer structure has not been synthesized yet, there are several theoretical studies that predict and investigate the physical properties of $2 \mathrm{D}$ monolayer $\mathrm{ZrS}_{3}$. Jin et al. predicted the possibility for exfoliation of monolayer $\mathrm{ZrS}_{3}$ from its bulk form by calculating the cleavage energy. ${ }^{85} \mathrm{It}$ was shown that low cleavage energy of $\mathrm{ZrS}_{3}$ favors a stable exfoliated monolayer structure. In addition, electronic-band structure calculations revealed that monolayer $\mathrm{ZrS}_{3}$ is an indirect-gap semiconductor with a bandgap of $1.90 \mathrm{eV}$. Moreover, it was reported that like other monolayer TMT, $\mathrm{ZrS}_{3}$ exhibits anisotropic conduction properties which were demonstrated by calculating the electron and hole effective masses. Li et al. studied the tunable electronic properties of
TMTs family including monolayer $\mathrm{ZrS}_{3} .{ }^{86}$ It was found that monolayer $\mathrm{ZrS}_{3}$ can undergo an indirect-to-direct bandgap transition as a function of applied tensile strain $(\varepsilon=2 \%)$. It was also pointed out that this tunable electronic structure provides opportunities for designing structures for applications in optoelectronics and flexible electronics.

Although its 1D NR form has not been synthesized yet, quasi-1D crystal of $\mathrm{ZrS}_{3}$ has been studied. Osada et al. investigated the phonon properties of few-layer quasi-1D form of $\mathrm{ZrS}_{3} .{ }^{87} \mathrm{~A}$ considerable downshift of the frequency of the $\mathrm{A}_{g}^{3}$ mode with decreasing number of layers and a slight downshift of the $\mathrm{A}_{g}^{5}$ mode were found which were explained through intrachain, interchain, and interlayer force constants using simple 1D lattice models. Thus, it was pointed out that the large shift of the $\mathrm{A}_{g}^{3}$ mode reflects the quasi-1D structure of $\mathrm{ZrS}_{3}$. Very recently, Pant et al. studied the strong dichroic emission in quasi-1D $\mathrm{ZrS}_{3}$ and found that the optical properties of few-layer $\mathrm{ZrS}_{3}$ are highly anisotropic as revealed by PL intensity variation with the direction of polarization. ${ }^{88} \mathrm{It}$ was pointed out that this observed PL variation with polarization has a strong similarity with conventional $1 \mathrm{D}$ materials with the difference that these 1D chains interact with each other in this structure.

\section{BLACKPHOSPHORUS NRs (bpNRs)}

Blackphosphorus, which was recently synthesized as a new member of the class of 2D materials, exhibits unique physical properties due to its anisotropic and puckered structure. Das et al. demonstrated the formation of few-nanometer-wide BPNRs with both zigzag and armchair edges with 
controlled structural modification of few layer blackphosphorus. ${ }^{89}$ Using a top-down method, it is possible to fabricate NR from suspended few-layer blackphosphorus flakes. The fabricated BPNRs were about $10 \mathrm{~nm}$ on width and few layers on thickness.

Even though the experimental studies on BPNRs are very limited, there are already important theoretical studies that analyze their physical properties. Zhang et al. studied the electronic properties of BPNRs having different widths and edge configurations and reported that BPNRs with armchair edges are semiconductors, while the zigzag BPNRs are metallic. ${ }^{90}$ For armchair BPNRs, it was shown that the bandgap decreases as the width of the NR increases. After $\mathrm{H}$-passivation of the edges, zigzag BPNRs become semiconducting, while the bandgap of the armchair BPNR gets larger than for bulk phosphorene. In addition, it was demonstrated that all the semiconducting NRs exhibit very large values of Seebeck coefficient (SC) and are tunable upon hydrogen passivation of the edges. Ding et al. also investigated the effect of edge passivation on the electronic structure of BPNRs. ${ }^{91}$ The edges of BPNRs are highly active and can be bonded easily with oxygen atoms and hydroxyl groups demonstrating that BPNRs can be easily oxidized. In addition, it was shown that a transition from semimetallic character to semiconducting can be achieved in both zigzag and armchair BPNRs upon oxidation.

Localized surface plasmon resonance (LSPR) is an optical phenomenon generated by light when it interacts with conductive smaller particles than the wavelength of incident light. Liu and Aydin investigated LSPR in monolayer of BPNRs and LSPR at mid-infrared and far-infrared wavelength regimes was demonstrated.$^{92}$ It was shown that due to the strong anisotropic in-plane properties, BPNRs can display polarization dependent anisotropic plasmonic response.

In another study, Sorkin and Zhang investigated the mechanical properties BPNRs under uniaxial tensile strain and reported that the deformation and failure of BPNRs are highly anisotropic. ${ }^{93}$ It was reported that BPNRs have a high failure strain $(\varepsilon=0.5)$ along the armchair direction with a low failure stress $(\sigma=50 \mathrm{GPa})$, while along the zigzag direction the failure occurs at early stages of tensile strain $(\varepsilon=0.1)$ but with a relatively high failure stress $(\sigma=100 \mathrm{GPa})$. For both directions, the value of the failure stress is sensitive to the width of the BPNRs, while the failure strain is unaffected.

\section{GROUP IV NRs}

The first synthesized 2D material is known as graphene which consists of $\mathrm{C}$ atoms that belong to the IV member in periodic table. After successful isolation of graphene from its bulk counterpart, graphite, researchers started to search for other group IV atoms that are able to form a 2D structure. Monolayer crystals of silicon, namely, silicene, germanene, and stanene, were first predicted theoretically to be buckled stable monolayers and then successfully synthesized on different substrates. ${ }^{94-99}$ Due to the same structural properties with graphene, these monolayers exhibit similar electronic properties with those of graphene such as possessing Dirac cone and thus, dimensional reduction in these materials gained a lot of interest as in the case of other 2D materials.

\section{A. GNRs' cousin: Silicene NRs}

Silicene, a 2D honeycomb structure of Si atoms with a buckled geometry, has been attracting great interest due to its physical properties such as possessing massless Dirac fermions and large spin-orbit coupling resulting in an intrinsic bandgap. 1D NRs of silicene have been also successfully synthesized.

Production of silicon NRs (SiNRs) on different substrates was performed in various experiments. In one of them, De Padova et al. synthesized silicon NRs (SiNRs) by depositing $\mathrm{Si}$ atoms on silver (110) surface under UHV conditions. ${ }^{100,101}$ The synthesized NRs have lengths of nanometers and about $1.6 \mathrm{~nm}$ of width with just $0.2 \mathrm{~nm}$ of height. Highresolution synchrotron radiation photoelectron spectroscopy measurements demonstrate a strong metallic character in these SiNRs. DFT calculations showed that NRs have a silicene like honeycomb structure on the silver substrate. Tchalala et al. experimentally observed self-assembled SiNRs on $\mathrm{Au}(110)-2 \times 1$ reconstructed surface. ${ }^{102}$ Low electron energy diffraction (LEED) showed a new superstructure which corresponds to SiNRs observed upon adsorption of $\mathrm{Si}$ atoms on $\mathrm{Au}(110)$ surface. STM and high-resolution photoemission spectroscopy measurements demonstrated that these SiNRs are flat and have widths of $1.6 \mathrm{~nm}$. These NRs show very similar structural properties with the ones synthesized on $\operatorname{Ag}(110)$ surface. De Padova et al. successfully synthesized multilayer of SiNRs on $\operatorname{Ag}(110)$ surface. ${ }^{103}$ It was shown by angle-resolved photoemission spectroscopy (ARPES) that the synthesized SiNRs have a gap of $0.56 \mathrm{eV}$ centered at $0.62 \mathrm{eV}$ below the Fermi level which differs from the one-atom-thick structure. The SiNRs were formed to have zigzag edges with hexagon apexes aligned along their lengths. Feng et al. ${ }^{104}$ recently studied the structure and quantum well states (QWS) in silicene NRs (SiNRs) which were grown on $\mathrm{Ag}(110)$ surface as performed by De Padova et al. (see Figs. 17-19). The structure of the synthesized SiNRs has a reconstructed armchair shape. Depending on the temperature, the width of the SiNRs can be $1 \mathrm{~nm}$ or $2 \mathrm{~nm}$ with increasing lengths. Pronounced quantum well states (QWS) were observed in SiNRs due to the confinement of quasiparticles perpendicular to the NR. The origin of QWS arises from the metallic states of SiNRs and was found to be substrate-independent.

Stiffness or flexibility of a material can be determined through its mechanical parameters. Topsakal and Ciraci investigated the mechanical properties of SiNRs in both elastic and plastic regimes under applied uniaxial tension. ${ }^{105}$ It was shown that the electronic and magnetic structures of SiNRs are very sensitive to the applied uniaxial strain. The calculated in-plane stiffness is about $51 \mathrm{~N} / \mathrm{m}$ which is very small compared with that of GNRs $(292 \mathrm{~N} / \mathrm{m})$ which indicates the larger flexibility of the SiNRs. Jing et al. investigated the mechanical properties of SiNRs under uniaxial tension and found that the Young modulus is strongly 

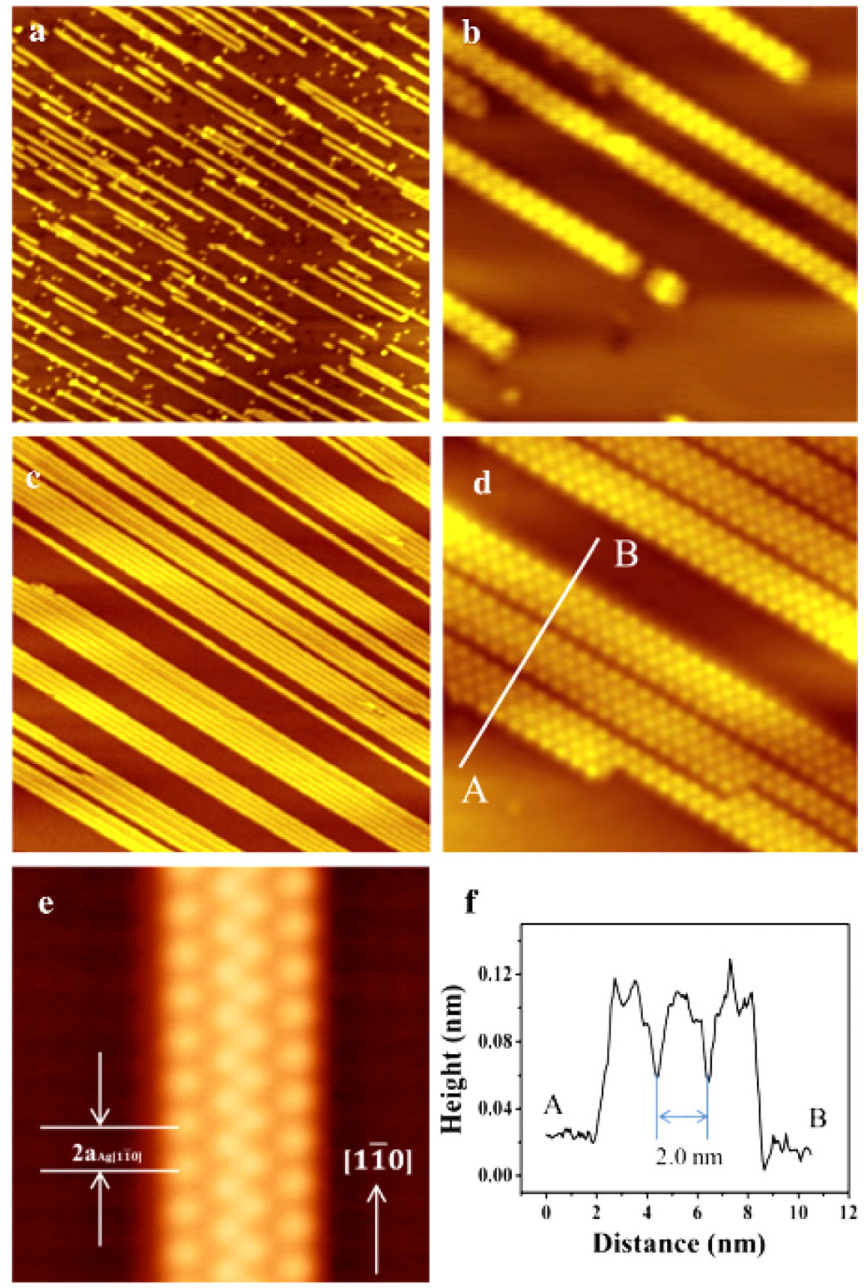

FIG. 17. (a) STM topographic image of silicon deposited on $\operatorname{Ag}(110)$ surface at room temperature. (b) High resolution STM image (taken at $15 \times$ $15 \mathrm{~nm}^{2}$ at $\mathrm{V}=1.1 \mathrm{~V}$ ) of the $1 \mathrm{~nm}$ wide SiNRs. Reprinted with permission from Feng et al., Surf. Sci. 645, 74 (2016). Copyright 2016 Elsevier.

affected by the chirality and the size of the SiNRs due to edge effects. ${ }^{106}$ It was reported that the Young modulus of the SiNRs increases as the size of the NR increases.

NRs forms of materials may exhibit edge-type- and with-dependent magnetic and electronic properties. Zhao and $\mathrm{Ni}$ studied the magnetic and electronic properties of SiNRs with a different edge structure which was called sawtooth-edged SiNRs (SSiNRs). ${ }^{107}$ It was found that SSiNRs are more stable than the zigzag-edged SiNRs and have FM spin-semiconductor character with an intrinsic gap between the majority and minority spin states. It was predicted that an applied small tensile strain can make the material more sensitive to an external electric field which can be used to tune its electronic properties. With all these findings, it was suggested that SSiNRs may have potential for applications in spintronic devices. Song et al. investigated the effect of the edge structure and the width of SiNRs on the structural and electronic properties. ${ }^{108}$ They considered both edge shapes, zigzag and armchair, with $\mathrm{H}$ passivation for SiNRs and found that an edge state appears in zigzag SiNRs at the Fermi level, $\mathrm{E}_{F}$, which cannot be observed in all armchair SiNRs. It was found that increasing the width of armchair
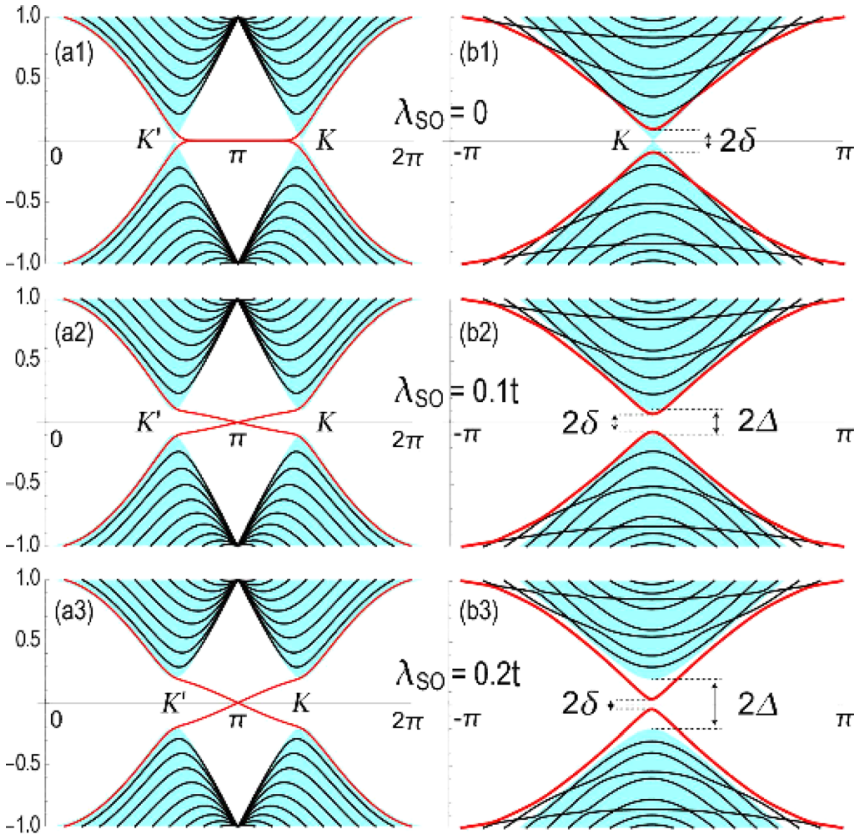

FIG. 18. Band structure of ((a1), (a2), and (a3)) zigzag and ((b1), (b2), and (b3)) armchair nanoribbons for width of $\mathrm{N}=16$. The vertical axis is the energy in units of $\mathrm{t}$ and the horizontal axis is the momentum $\mathrm{k}$. The cyan region (red curve) represents the band of the bulk (edge). The bandgap of the bulk (edge) is denoted by $2 \Delta(2 \delta)$ which increases (decreases) as $\lambda_{S O}$ increases. Reprinted with permission from M. Ezawa and N. Nagaosa, Phys. Rev. B 88, 121401 (2013). Copyright 2013 American Physical Society.

SiNRs, both an oscillating behavior and a periodic feature of $\Delta_{3 n}>\Delta_{3 n+1}>\Delta_{3 n+2}$ for integer $\mathrm{n}$ were predicted.

Spin-dependent magnetic properties in crystals are a determinative property for their applications in spintronic devices. Fu et al. studied the spin-dependent Seebeck effect (SDSE) in H-terminated zigzag-edged SiNRs and found that
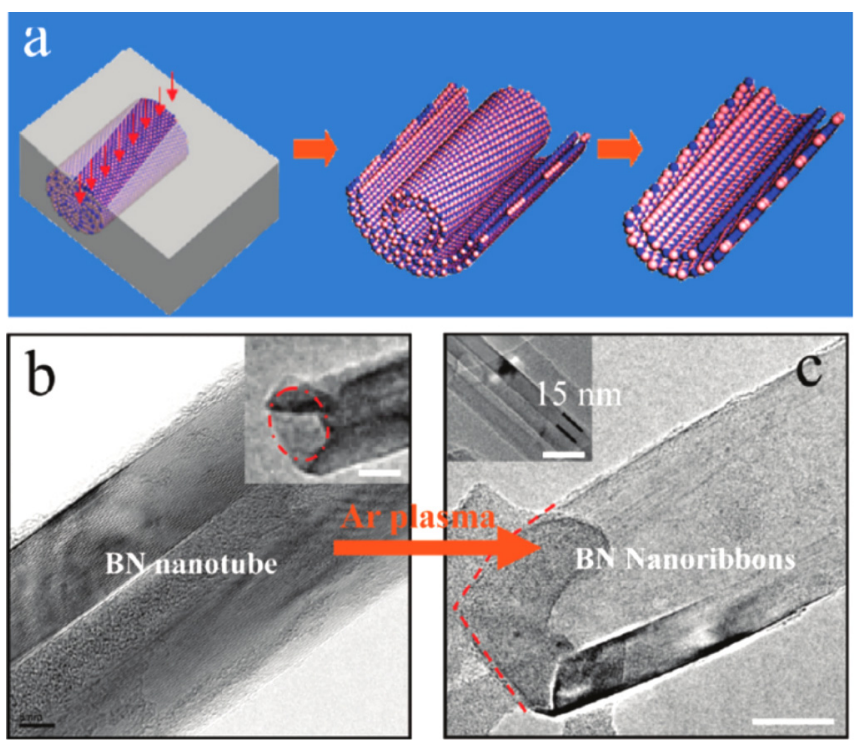

FIG. 19. Unwrapping of MWBNNTs. (a) Schematic representation of the unwrapping processes induced by plasma etching, and exfoliating of tube walls to form BNNRs. (b) and (c) Morphology transition typical TEM images of the products before and after etching. The inset in (b) reveals the open and nearly circular end of the initial tube, and the inset in (c) shows four formed ribbons; the scale bars are $20 \mathrm{~nm}$. Reprinted with permission from Zeng et al., Nano Lett. 10, 5049 (2010). Copyright 2010 American Physical Society. 
applying temperature difference between source and drain results in oppositely flowing spin-up and spin-down currents indicating thermal spin currents in zigzag SiNRs. ${ }^{109}$ Creating opposite spin currents reduces the conducting electron current in the material and thus, the related Joule heating is much suppressed which indicates potential applications of zigzag SiNRs in low-power-consumption technology. An et al. studied spin-polarized currents in SiNRs by applying local exchange field and reported that upon applying a weak local exchange field parallel to the surface of SiNRs on one of the edges of the NR, a gap is induced in the corresponding edge states while another pair of gapless edge states with opposite spin are still protected by time-reversal symmetry. ${ }^{110}$ It was pointed out that spin-polarized current can be obtained in the absence of Rashba spin-orbit coupling and in the presence of a very weak exchange field. Saari et al. showed that in terms of an inhomogeneous electric field, conducting channels can be localized in SiNRs. ${ }^{111}$ In the same way, it is possible to break the spin degeneracy of these conducting channels. They mentioned that silicene type group IV elements can be of potential for spintronic applications with manipulated spin currents. Moreover, tunability of spin and charge transport in SiNRs having zigzag edge shape were investigated by Shakouri et al. ${ }^{112}$ They found that the band structure of zigzag SiNR lacks spin inversion symmetry in the presence of intrinsic SOC in combination of an external electric field and an exchange field. Moreover, for certain energy ranges of the incoming electrons, SiNR was found to possess a controllable high-efficiency spin polarizing behavior. $^{113}$

Very recently, Zare et al. studied the topological phase and dependency of Ruderman-Kittel-Kasuya-Yosida (RKKY) interaction on the edge states in zigzag SiNRs. ${ }^{114} \mathrm{It}$ was found that from the band dispersion of zigzag SiNRs, topological phase transition can be investigated in detailed by using RKKY interaction. It was shown that the exchange coupling is significantly enhanced due to the zero-energy edge states of the zigzag SiNRs. Moreover, it was revealed that three different magnetic phases, spiral, FM, and AFM, can exist under different values of an external electric field for a system of two magnetic impurities which are located on the edges of zigzag SiNR. In addition, Ezawa and Nagaosa studied the edge channels of SiNRs and found that the behavior of the helical edge channels (HECs) strongly depends on the shape of the edge. ${ }^{115}$

\section{B. NRs of germanene and stanene}

Like monolayer silicene, the 2D form of germanium, germanene, has been synthesized as a buckled monolayer. Due to its relatively high spin-orbit gap, it was considered as an ideal candidate to exhibit the quantum spin Hall effect at experimentally accessible temperatures.

NRs of germanene have been proposed theoretically. Pang et al. investigated the electronic and magnetic properties of both pristine and chemically doped GeNRs and found that a single $\mathrm{B}$ or $\mathrm{N}$ substitution can induce a transition from semiconducting to metal in armchair GeNRs. ${ }^{116}$ In addition, a single $\mathrm{B}$ or $\mathrm{N}$ doping turns $\mathrm{AFM}-$ semiconducting zigzag
GeNRs into FM-semiconductor. Bayani et al. studied the optical properties of hydrogenated GeNRs and the properties of $\mathrm{H}$ sensitive FET based on GeNRs. ${ }^{117}$ The stable structure, binding energy, and depleted charge in armchair GeNRs for different amounts of $\mathrm{H}_{2}$ concentrations were obtained and it was found that increasing the $\mathrm{H}_{2}$ concentration also increases the binding energy and the transferred amount of charge. In addition, for the armchair GeNR with 5 chain width, the effect of $\mathrm{H}_{2}$ concentration on the transport properties of GeNRs was investigated and found that under the same applied bias voltage, current through the GeNR increases with increasing $\mathrm{H}_{2}$ concentration. To obtain the optical properties, the dielectric functions were calculated for parallel and perpendicular polarizations and the dielectric function was found to be anisotropic, and the dielectric constant decreases as the $\mathrm{H}_{2}$ concentration increases. Wang et al. predicted that in H-passivated zigzag edge SiNRs and GeNRs, half-metallicity can be induced by applying an in-plane electric field. ${ }^{118}$ It was reported that H-passivated zigzag edge SiNRs have a longer spin relaxation time and spin-diffusion length with larger magnetic stability when compared to $2 \mathrm{D}$ silicene.

Stanene, consisting only of $\mathrm{Sn}$ atoms, is a 2D material having many physical properties similar to those of silicene and germanene which was recently synthesized on $\mathrm{Bi}_{2} \mathrm{Te}_{3}$ (111). ${ }^{119}$ The 1D NR form of stanene has not been synthesized experimentally but was already investigated theoretically.

1D NR form of Sn (SnNR) has not been synthesized yet but theoretical investigations were achieved on its fundamental properties. Xiong et al. studied the electronic and magnetic properties of SnNRs and reported that armchair stanene NRs (ASnNRs) are NM semiconductors, while zigzag SnNRs (ZSnNRs) possess AFM semiconducting ground state. $^{120}$ In addition, for bandgap of ASnNRs, a periodic oscillating behavior with the width was found. However, an inverse relation was found between the bandgap of ZSnNRs and the width. Investigating $\mathrm{I}-\mathrm{V}$ characteristic of materials is important for realizing its bandgap and device performance. van den Broek et al. investigated the current-voltage characteristic of armchair stanene NRs (ASnNRs) and found that without any strain SnNRs carry higher currents for low biases when compared with the other group-IV NRs. ${ }^{121}$ It was analyzed that the bandgap of ASnNRs depends on the width of the ribbon. In addition, it was shown that for a certain width the SnNRs exhibit comparable contact resistance independent of the increasing $s p^{3}$ hybridization character. In another study, van den Broek and his co-workers studied the electronic structure and transport properties of ASnNRs and reported that due to the $s p^{2}-s p^{3}$ hybridization the electronic structure can be tuned by applying out-of-plane electric field and in-plane uniaxial strain. ${ }^{122}$ It was reported that since stanene has the largest buckling among the group-IV monolayers, these external effects decrease the current for SnNRs with about $50 \%$ in $\mathrm{I}-\mathrm{V}$ characteristic. It was also shown that when the polarization energy becomes comparable to the quantum confinement bandgap, a bandgap is induced and the effective mass increases. In addition to these studies, edge states in both hydrogen terminated and bare zigzag SnNRs 
(ZSnNRs) were investigated by Hattori et al. Without hydrogen terminations, the energy spectra of multi-orbital model and single-orbital model are different for low buckled ZSnNRs. ${ }^{123}$ For the hydrogenated case, it was revealed that due to the strong spin-orbit interaction the degeneracy of the edge states is removed and helical edge states with dispersion appear.

\section{NRs OF GROUP III-V COMPOUNDS}

\section{A. 1D form of white graphene: $h$-boron nitride NRs (BNNRs)}

Following the successful synthesis of graphene, synthesis of 2D monolayer form of hexagonal boron nitride (h-BN) was also achieved. ${ }^{124}$ 2D monolayer h-BN has attracted great attention due to its various outstanding properties such as high thermal conductivity, low dielectric constant, chemical inertness, and high mechanical strength which make this material important for a wide range of applications.

Like its 2D form, 1D NRs of h-BN were also widely studied. Chen et al. fabricated BN hollow NRs (BNHNRs) by using ZnS NR as a template since it can be synthesized easily by CVD. ${ }^{125}$ The fabricated BNHNRs have high crystallinity and demonstrate an ultraviolet cathodoluminescence (CL) emission at $5.33 \mathrm{eV}$ which is important for applications in optoelectronics. In another study, Zeng et al. produced few- and single-layer BNNRs, mostly with zigzag edges, by unwrapping multiwalled $\mathrm{BN}$ nanotubes (MWBNNTs) through plasma etching. ${ }^{126}$ It was found that the synthesized NRs possess semiconducting behavior due to vacancy defects and doping-like conducting edge states. It was also shown that the produced BNNRs have mainly N-terminated zigzag edges exhibiting high currents of $2 \mu \mathrm{A}$ under a applied voltage of $9 \mathrm{~V}$ and a high conductance of $104 \mathrm{~S} / \mathrm{m}$. Furthermore, ab-initio calculations demonstrated that experimentally observed surface vacancies are the driving sources for the distinct carrier transport in BNNRs. Wei et al. studied substitutional C-doping in BNNRs with in situ electron beam irradiation in an energy-filtering $300 \mathrm{kV}$ HRTEM. ${ }^{127}$ It was shown that $\mathrm{C}$ substitutions preferentially occur at the sites which are more vulnerable to electron beam irradiation like the edges of the BNNRs. In addition, it was observed that BNNRs can be transformed from an insulator to a conductor upon $\mathrm{C}$ doping in agreement with the previous theoretical predictions.

Especially after synthesis of BNNRs, theoretical studies have gained a lot of interest to analyze and propose unknown physical properties of BNNRs. Samarakoon and Wang investigated half-metallicity property in hydrogenated BNNRs and found that zigzag-edged BNNRs can possess intrinsic half-metallic feature upon full hydrogenation. ${ }^{128}$ In addition, this half-metallic behavior was reported to be tunable under applied transverse electric bias which is a promising route for spintronic applications. Similar results were also found by Topsakal et al. ${ }^{129}$ They found that armchair BNNRs are NM semiconductors, while the one with zigzag edges are metallic but they can also display FM semiconducting behavior upon hydrogen passivation of both edges. In addition, it was shown that the bandgap of armchair BNNRs can be widened upon passivation of $\mathrm{B}$ and $\mathrm{N}$ with $\mathrm{H}$ atoms. Furthermore, it was pointed out that magnetic and electronic properties of zigzag BNNRs strongly depend on edge passivation whether the B or N edge is saturated with $\mathrm{H}$. In another study, Lopez-Bezanilla et al. investigated electronic and magnetic properties of BNNRs under edge functionalization with different types of atoms. ${ }^{130}$ It was shown that in contrast to $\mathrm{H}$ passivated NM semiconducting zigzag BNNRs, Oxygen $(\mathrm{O})$ edge-terminated zigzag BNNRs can have two energetically degenerate FM metallic ground states on the B edges. However, the $\mathrm{S}$ edge terminated NRs were found to have NM metallic ground state. It was suggested that $\mathrm{O}$ and $S$ functionalizations play crucial roles in zigzag BNNRs for the use in electronic devices. In addition to these types of atoms, properties of fluor passivated zigzag edge BNNRs were studied by Zeng et al. ${ }^{131}$ It was demonstrated that the transition from half-metallic character to the semiconducting one can be observed in zigzag BNNRs by fluorination at different sites or by tuning the amount of fluorination. In addition, it was reported that 8-chain wide zigzag BNNRs with 1 and $8 \mathrm{~F}$ atoms possess perfect spin-filtering effect and negative differential resistance (NDR) behavior.

In all of the materials, it is very common to have defective structures while they are being synthesized. Theoretical investigations demonstrate that existence of such defects in a crystal may affect the magnetic and electronic properties of the material. Li et al. studied the effect of line defects on the electronic and magnetic properties of BNNRs. ${ }^{132}$ It was found that line-defect-embedded zigzag BNNRs whose edges are chemically homogeneous and B-terminated are AFM semiconductors in their ground state. However, those with N-terminated edges were reported to be metallic with both AFM and FM states. In addition, line-defect-embedded zigzag BNNRs with hydrogen passivation were found to be NM semiconductors with notably reduced bandgap which occurs due to the impurity states. Qi et al. investigated the piezoelectric properties of BNNRs and reported that the bandgap of zigzag-edged BNNRs can be tuned under uniaxial tensile strain. ${ }^{133}$ In addition, it was shown that due to the structural asymmetry and $\mathrm{H}$ passivation, sizable dipole moment and piezoelectric effect can be found in these NRs.

\section{B. h-AIN-NRs: Edge dependent magnetism}

Although NRs of Aluminum nitride (AlNNRs) have not been synthesized yet, there are several theoretical studies on this structure. In one of them, Zhang et al. investigated the effects of the dangling bond on the electronic and magnetic properties of AlNNRs. ${ }^{134}$ It was found that the dangling bonds in zigzag AlNNRs result in a magnetic semimetallic ground state with bare $\mathrm{N}$ edge, while they result in a magnetic metallic ground state for NRs with bare $\mathrm{Al}$ edge. However, for armchair AlNNRs with bare $\mathrm{N}$ or Al edges, dangling bonds cause a magnetic semiconducting ground state. It was also shown that due to the number of dangling bonds in zigzag AlNNRs which are half of those in armchair AlNNRs, the magnetic moment is also half of the one of armchair AlNNRs. Du et al. studied the structural and electronic properties of zigzag AlNNRs and reported that 
H-terminated zigzag AlNNRs having width of 10 chains are NM semiconductors with indirect bandgap. ${ }^{135}$ In addition, it was shown that an external transverse electric field decreases monotonically the bandgap. In another study, LopezBezanilla et al. investigated the effect of self-doping on the half-metallicity of AlNNRs and reported that this behavior can be tuned into fully metallic or semiconducting depending on the application of an external electric field or uniaxial strain. ${ }^{136}$ It was shown that as the uniaxial strain is varied from compressive to tensile, a spin-resolved selective selfdoping occurs which increases the half-metallicity of the NRs. In addition to these theoretical characterization studies, the effects of doping were also investigated. The structural and electronic properties of C-chain doped AlNNRs were investigated ${ }^{137,138}$ and reported that single C-chain doped AlNNRs, with both zigzag and armchair edges, are still NM semiconductors but the $\mathrm{C}$-chain reduces the bandgap. In addition, for zigzag AlNNRs, C-chain doping can change the direct character of the band structure to indirect one independent of its position.

\section{SUPERSTRUCTURES OF NRS}

In addition to the analysis of fundamental properties of NRs, construction of superlattices by using different types of NRs was first predicted by Sahin et al. ${ }^{139}$ (see Fig. 20) It was reported that a type-I band alignment can be induced due to the states which are confined to the GaN part of the heterostructure. Chen et al. also investigated the electronic properties of gallium nitride NRs (GaNNRs) and possible AlN/ GaN NR heterojunctions. ${ }^{140}$ It was found that the bandgap of GaNNRs is sensitive to the width of the ribbon and decreases monotonically with the increase in the width. The ribbons with zigzag edge are indirect semiconductors, while the ones with armchair edge are direct gap semiconductors. It was shown that for AlN/GaN NRs the bandgap increases monotonically with the increase of the AlN concentration in the heterojunction. Dai et al. also studied the same structures and reported similar results. ${ }^{141}$ Chandiramouli studied the transport properties of gallium arsenide NRs (GaAsNRs) based molecular devices and reported that under applied bias voltage the peak maximum in both valence and conduction bands increases. ${ }^{142}$ It was shown that the electron density is mostly localized on the As sites through GaAsNR and thus, the transmission spectrum provides the transmission of electrons at different energy intervals across GaAsNR.

Heterostructures of NRs can be obtained especially using structurally similar single layer materials such as $\mathrm{MoS}_{2}-\mathrm{WS}_{2}$, Si-Ge-Sn, or Graphene-hBN. Randhawa et al. studied the thermoelectric transport properties of hybrid SiGe NRs. ${ }^{143}$ The value for the thermoelectric figure of merit of hybrid Si-Ge NRs increases with the number of interfaces used in the hybrid NR. This was shown especially for Si-GeSi-Ge-Si NR (with 4 interfaces) and Si-Ge-Si NR (with 2 interfaces) that the thermoelectric figure of merit of the first hybrid NR was found to be enhanced as compared with that of the second one.

There are also several studies on hybrid NRs of B, N, and $\mathrm{C}$ atoms. Seol and Guo studied the bandgap tuning in

\section{(a) GaN/AIN Superlattice}
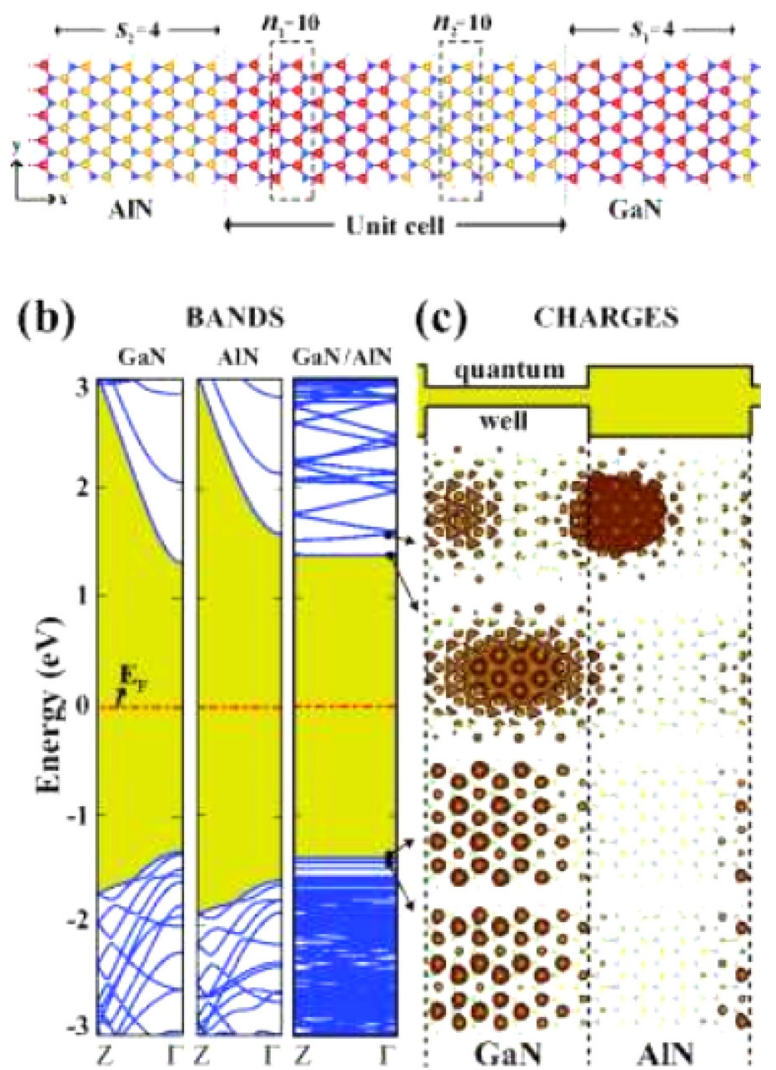

FIG. 20. (a) A superlattice formed by periodically repeating heterostructure of armchair NRs of GaN-AlN compounds. Red, yellow, blue, and small balls represent $\mathrm{Ga}, \mathrm{Al}, \mathrm{N}$, and $\mathrm{H}$ atoms, respectively. (b) Energy band structures of constituent GaNNRs, AlNNRs, and resulting superlattice. The bandgaps are shaded by yellow. (c) Band decomposed isosurface charge densities for lowest two conduction band and highest two valence bands. Reprinted with permission from Sahin et al., Phys. Rev. B 80, 155453 (2009). Copyright 2009 American Physical Society.

AGNRs which are confined by BNNRs. ${ }^{144}$ It was found that H-terminated AGNRs have a nearly zero bandgap for width of $3 N+2$, while the ones confined by BNNRs exhibit considerable bandgap. It was pointed out that this fact originates from the change in potential energy of $\mathrm{C}$ atoms in the edge of AGNR that is close to $\mathrm{B}$ and $\mathrm{N}$ atoms in BNNR. In another study, He et al. obtained a transition from insulator to metallic character in hybridized graphene and boron nitride NRs. ${ }^{145}$ In addition, the electronic and transport properties of zigzag-edged BNNR and GNR were investigated. The bandgap can be tuned by changing the width of the ZGNR and transition from insulator to semiconductor, halfsemimetal, and metal can be induced for certain number of zigzag BNNR and ZGNR atoms.

As an example of heterostructures of TMDs, both 1D and 2D forms of the $\mathrm{MoS}_{2}-\mathrm{WS}_{2}$ were investigated. Kang et al. investigated the band alignment tunability and charge localization of heterostructures of $\mathrm{MoS}_{2}-\mathrm{WS}_{2}{ }^{146}$ They found that under applied tensile strain on $\mathrm{WS}_{2}$ region, a type-II to type-I band alignment transition can take place. In addition, it was shown that the presence of the grain boundary introduces localized in-gap states. Moreover, they reported that the carrier localization is tunable under varying of the width of 
the constituents of the superlattice. Recently, Zhang et al. theoretically predicted the thermoelectric properties of $\mathrm{MoS}_{2}-\mathrm{WS}_{2}$ based hybrid $\mathrm{NRs}^{147}$ and showed that the hybrid NRs exhibit higher efficiency of energy conversion than their individual NRs due to the fact that the $\mathrm{MoS}_{2} / \mathrm{WS}_{2}$ interface reduces lattice thermal conductivity more than the electron transport. Furthermore, it was revealed that depending on the number of the interfaces between $\mathrm{MoS}_{2} / \mathrm{WS}_{2} \mathrm{NRs}$, a figure of merit ZT as high as 5.5 can be achieved at a temperature of $600 \mathrm{~K}$.

\section{SUMMARY}

In this study, we reviewed the synthesis techniques, characterization methods, fundamental properties, and stateof-the-art applications of nanoribbons of ultrathin twodimensional crystal structures.

Here, we show that synthesis and usage of these NR materials are important due to their controllable synthesis processes, length, width, and edge shape and edge passivation possibilities. GNRs are pointed out as 1D structures with ultranarrow width and thickness. In addition, width and edge-shape dependent magnetic and electronic properties are promising for their optoelectronic applications. Zigzag edge $\mathrm{MoS}_{2}$ NRs are reported to exhibit width-independent physical properties with suitable bandgap for various applications. As anisotropic materials BPNRs and $\mathrm{ReS}_{2}$ NRs have unique properties such as growing time dependent morphology for $\mathrm{ReS}_{2}$ NRs and very high Seebeck coefficient for BPNRs. As a member of TMTs, $\mathrm{TiS}_{3}$ NRs are mentioned to have high electron mobility and edge-dependent electronic properties. Moreover, from the group of $\mathrm{C}$ element, SiNRs are reported to be very flexible materials and temperature-dependent width in synthesis process is pointed out as an important remark. Through the same group in periodic table, SnNRs are announced to have high currents for low voltages without any strain. As a member of group III-V compounds, NRs of h-BN are known to exhibit edge-passivation-dependent magnetic and electronic properties. Furthermore, recent theoretical investigations reveal the existence of superlattices of NRs which addresses the enhanced electronic, thermal, and transport properties.

Our review demonstrates that size, edge-type, and thickness in 1D NRs of novel 2D materials can strongly affect their electronic, magnetic, optical, and mechanical properties. Recent advances in nanoscale material research have revealed that $1 \mathrm{D}$ ultra-thin nanoribbons are not only essential for demonstration of enhanced quantum effects which promise some interesting discoveries but also important due to their possible use in nanotechnology applications.

\section{ACKNOWLEDGMENTS}

Computational resources were provided by TUBITAK ULAKBIM, High Performance and Grid Computing Center (TR-Grid e-Infrastructure). H.S. acknowledges the support from Bilim Akademisi-The Science Academy, Turkey under the BAGEP program. R.T.S. acknowledges the support from TUBITAK through Project No. 114F397. F.M.P. was supported by the Flemish Science Foundation (FWO-Vl) and the Methusalem program.
${ }^{1}$ K. S. Novoselov, A. K. Geim, S. V. Morozov, D. Jiang, Y. Zhang, S. V. Dubonos, I. V. Grigorieva, and A. A. Firsov, Science 306, 666 (2004).

${ }^{2}$ C. G. Lee, X. D. Wei, J. W. Kysar, and J. Hone, Science 321, 385 (2008).

${ }^{3}$ K. S. Novoselov, A. K. Geim, S. V. Morozov, D. Jiang, M. I. Katsnelson, I. V. Grigorieva, S. V. Dubonos, and A. A. Firsov, Nature (London) 438, 197 (2005).

${ }^{4}$ A. A. Balandin, S. Ghosh, W. Bao, I. Calizo, D. Teweldebrhan, F. Miao, and C. N. Lau, Nano Lett. 8, 902 (2008).

${ }^{5}$ X. Li, X. Wang, L. Zhang, S. Lee, and H. Dai, Science 319, 1229 (2008).

${ }^{6}$ J. Cai, P. Ruffieux, R. Jaafar, M. Bieri, T. Braun, S. Bankenburg, M.

Muoth, A. P. Seitsonen, M. Saleh, X. Feng, K. Mllen, and R. Fasel,

Nature (London) 466, 470 (2010).

${ }^{7}$ T. H. Vo, M. Shekhirev, D. A. Kunkel, M. D. Morton, E. Berglund, L. Kong, P. M. Wilson, P. A. Dowben, A. Enders, and A. Sinitskii, Nat. Commun. 5, 3189 (2014).

${ }^{8}$ A. Kimouche, M. M. Ervasti, R. Drost, S. Halonen, A. Harju, P. M. Joensuu, J. Sainio, and P. Liljeroth, Nat. Commun. 6, 10177 (2015).

${ }^{9}$ J. Bai, X. Duan, and Y. Huang, Nano Lett. 9, 2083 (2009).

${ }^{10}$ P. Ruffieux, S. Wang, B. Yang, C. Sanchez-Sanchez, J. Liu, T. Dienel, L. Talirz, P. Shinde, C. A. Pignedoli, D. Passerone, T. Dumslaff, X. Feng, K. Mllen, and R. Fasel, Nature (London) 531, 489 (2016).

${ }^{11}$ L. C. Campos, V. R. Manfrinato, J. D. Sanchez-Yamagishi, J. Kong, and P. Jarillo-Herrero, Nano Lett. 9, 2600 (2009).

${ }^{12}$ L. Weng, L. Zhang, Y. P. Chen, and L. P. Rokhinson, Appl. Phys. Lett. 93, 093107 (2008).

${ }^{13}$ S. Masubuchi, M. Ono, K. Yoshida, K. Hirakawa, and T. Machida, Appl. Phys. Lett. 94, 082107 (2009).

${ }^{14}$ D. V. Kosynkin, W. Lu, A. Sinitskii, G. Pera, Z. Sun, and J. M. Tour, ACS Nano 5, 968 (2011).

${ }^{15}$ L. Jiao, L. Zhang, X. Wang, G. Diankov, and H. Dai, Nature 458, 877 (2009).

${ }^{16}$ L. Jiao, X. Wang, G. Diankov, H. Wang, and Hi Dai, Nat. Nanotechnol. 5, 321 (2010).

${ }^{17}$ M. Sprinkle, M. Ruan, Y. Hu, J. Hankinson, M. Rubio-Roy, B. Zhang, X. Wu, C. Berger, and W. A. de Heer, Nat. Nanotechnol. 5, 727 (2010).

${ }^{18}$ R. M. Jacobberger, B. Kiraly, M. Fortin-Deschenes, P. L. Levesque, K. M. McElhinny, G. J. Brady, R. R. Delgado, S. S. Roy, A. Mannix, M. G. Lagally, P. G. Evans, P. Desjardins, R. Martel, M. C. Hersam, N. P. Guisinger, and M. S. Arnold, Nat. Commun. 6, 8006 (2015).

${ }^{19}$ P. Han, K. Akagi, F. F. Canova, R. Shimizu, H. Oguchi, S. Shiraki, P. S. Weiss, N. Asao, and T. Hitosugi, ACS Nano 9, 12035 (2015).

${ }^{20}$ C. Stampfer, J. Guttinger, S. Hellmuller, F. Molitor, K. Ensslin, and T. Ihn, Phys. Rev. Lett. 102, 056403 (2009).

${ }^{21}$ H. Lee, Y.-W. Son, N. Park, S. Han, and J. Yu, Phys. Rev. B 72, 174431 (2005).

${ }^{22}$ Y.-W. Son, M. L. Cohen, and S. G. Louie, Nature 444, 347 (2006).

${ }^{23}$ E.-J. Kan, Z. Li, J. Yang, and J. G. Hou, J. Am. Chem. Soc. 130, 4224 (2008).

${ }^{24}$ Y.-W. Son, M. L. Cohen, and S. G. Louie, Phys. Rev. Lett. 97, 216803 (2006).

${ }^{25}$ L. Yang, C.-H. Park, Y.-W. Son, M. L. Cohen, and S. G. Louie, Phys. Rev. Lett. 99, 186801 (2007).

${ }^{26}$ A. A. Shylau, S. M. Badalyan, F. M. Peeters, and A. P. Jauho, Phys. Rev. B 91, 205444 (2015).

${ }^{27}$ F. Cervantes-Sodi, G. Csanyi, S. Piscanec, and A. C. Ferrari, Phys. Rev. B 77, 165427 (2008).

${ }^{28}$ T. Wassmann, A. P. Seitsonen, A. M. Saitta, M. Lazzeri, and F. Mauri, Phys. Rev. Lett. 101, 096402 (2008).

${ }^{29}$ B. Biel, X. Blase, F. Triozon, and S. Roche, Phys. Rev. Lett. 102, 096803 (2009).

${ }^{30}$ P. Scuracchio, S. Costamagna, F. M. Peeters, and A. Dobry, Phys. Rev. B 90, 035429 (2014).

${ }^{31}$ Z. Z. Zhang, Z. H. Wu, K. Chang, and F. M. Peeters, Nanotechnology 20, 415203 (2009)

${ }^{32}$ M. D. Petrovic and F. M. Peeters, Phys. Rev. B 91, 035444 (2015).

${ }^{33}$ S. Droscher, H. Knowles, Y. Meir, K. Ensslin, and T. Ihn, Phys. Rev. B 84, 073405 (2011).

${ }^{34}$ B. Huang, Q. Yan, G. Zhou, J. Wu, B.-L. Gu, and W. Duan, Appl. Phys. Lett. 91, 253122 (2007).

${ }^{35}$ H. Ren, Q.-X. Li, Y. Luo, and J. Yang, Appl. Phys. Lett. 94, 173110 (2009).

${ }^{36}$ D. C. Elias, P. R. Nair, T. M. Mohiuddin, S. V. Morozov, P. Blake, M. P. Halsall, A. C. Ferrari, D. W. Boukhvalov, M. I. Katsnelson, A. K. Geim, and K. S. Novoselov, Science 323, 610 (2009). 
${ }^{37}$ H. Sahin, C. Ataca, and S. Ciraci, Phys. Rev. B 81, 205417 (2010).

${ }^{38}$ B. Radisavljevic, A. Radenovic, J. Brivio, V. Giacometti, and A. Kis, Nat. Nanotechnol. 6, 147 (2011).

${ }^{39}$ K. F. Mak, C. Lee, J. Hone, J. Shan, and T. F. Heinz, Phys. Rev. Lett. 105, 136805 (2010).

${ }^{40}$ Q. Li, E. C. Walter, W. E. van der Veer, B. J. Murray, J. T. Newberg, E. W. Bohannan, J. A. Switzer, J. C. Hemminger, and R. M. Penner, J. Phys. Chem. B 109, 3169 (2005).

${ }^{41}$ X. Liu, T. Xu, X. Wu, Z. Zhang, J. Yu, H. Qiu, J.-H. Hong, C.-H. Jin, J.-X. Li, X.-R. Wang, L.-T. Sun, and W. Guo, Nat. Commun. 4, 1776 (2013)

${ }^{42}$ Z. Wang, H. Li, Z. Liu, Z. Shi, J. Lu, K. Suenaga, S.-K. Joung, T. Okazaki, Z. Gu, J. Zhou, Z. Gao, G. Li, S. Sanvito, E. Wang, and S. Iijima, J. Am. Chem. Soc. 132, 13840 (2010).

${ }^{43}$ H. Liu, J. Gu, and P. D. Ye, IEEE Electron Device Lett. 33, 1273 (2012).

${ }^{44}$ T. F. Jaramillo, K. P. Jorgensen, J. Bonde, J. H. Nielsen, S. Horch, and I. Chorkendorff, Science 317, 100 (2007).

${ }^{45}$ D. Y. Chung, S.-K. Park, Y.-H. Chung, S.-H. Yu, D.-H. Lim, N. Jung, H. C. Ham, H.-Y. Park, Y. Piao, S. J. Yoo, and Y.-E. Sung, Nanoscale 6, 2131 (2014).

${ }^{46}$ Y. Li, Z. Zhou, S. Zhang, and Z. Chen, J. Am. Chem. Soc. 130, 16739 (2008).

${ }^{47}$ C. Ataca, H. Sahin, E. Akturk, and S. Ciraci, J. Phys. Chem. C 115, 3934 (2011).

${ }^{48}$ K. Dolui, C. D. Pemmaraju, and S. Sanvito, ACS Nano 6, 4823 (2012).

${ }^{49}$ F. Ouyang, Z. Yang, X. Ni, N. Wu, Y. Chen, and X. Xiong, Appl. Phys. Lett. 104, 071901 (2014).

${ }^{50}$ S. Yang, D. Li, T. Zhang, Z. Tao, and J. Chen, J. Phys. Chem. C 116, 1307 (2012)

${ }^{51}$ Y. Li, D. Wu, Z. Zhou, C. R. Cabrera, and Z. Chen, J. Phys. Chem. Lett. 3, 2221 (2012)

${ }^{52}$ D. D. Fan, H. J. Liu, L. Cheng, P. H. Jiang, J. Shi, and X. F. Tang, Appl. Phys. Lett. 105, 133113 (2014).

${ }^{53}$ X. Liu, G. Zhang, Q.-X. Pei, and Y.-W. Zhang, Appl. Phys. Lett. 103, 133113 (2013)

${ }^{54}$ T.-H. Liu, Y.-C. Chen, C.-W. Pao, and C.-C. Chang, Appl. Phys. Lett. 104, 201909 (2014).

${ }^{55}$ Z. Wang, K. Zhao, H. Li, Z. Liu, Z. Shi, J. Lu, K. Suenaga, S.-K. Joung, T. Okazaki, Z. Jin, Z. Gu, Z. Gao, and S. Iijima, J. Mater. Chem. 21, 171 (2011).

${ }^{56}$ C. Nethravathi, A. A. Jeffery, M. Rajamathi, N. Kawamoto, R. Tenne, D. Golberg, and Y. Bando, ACS Nano 7, 7311 (2013).

${ }^{57}$ H. Zhang, X.-B. Li, and L.-M. Liu, J. Appl. Phys. 114, 093710 (2013).

${ }^{58}$ F. Ouyang, X. Ni, Z. Yang, Y. Chen, X. Zheng, and X. Xiong, J. Appl. Phys. 114, 213701 (2013).

${ }^{59}$ D. G. Kvashnin, L. Yu. Antipina, P. B. Sorokin, R. Tenne, and D. Golberg, Nanoscale 6, 8400 (2014).

${ }^{60}$ F. Lopez-Urias, A. L. Elias, N. Perea-Lopez, H. R. Gutierrez, M. Terrones, and H. Terrones, 2D Mater. 2, 015002 (2015).

${ }^{61}$ S. Tongay, H. Sahin, C. Ko, A. Luce, W. Fan, K. Liu, J. Zhou, Y. S. Huang, C. H. Ho, J. Y. Yan, D. F. Ogletree, S. Aloni, J. Ji, S. S. Li, J. B. Li, F. M. Peeters, and J. Q. Wu, Nat. Commun. 5, 3252 (2014).

${ }^{62}$ S. Yang, S. Tongay, Y. Li, Q. Yue, J.-B. Xia, S.-S. Li, J. Li, and S.-H. Wei, Nanoscale 6, 7226 (2014).

${ }^{63}$ S. Yang, C. Wang, H. Sahin, H. Chen, Y. Li, S.-S. Li, A. Suslu, F. M. Peeters, Q. Liu, J. Li, and S. Tongay, Nano Lett. 15, 1660 (2015).

${ }^{64}$ X. He, F. Liu, P. Hu, W. Fu, X. Wang, Q. Zeng, W. Zhao, and Z. Liu, Small 11, 5423 (2015).

${ }^{65}$ Z. G. Yu, Y. Cai, and Y.-W. Zhang, Sci. Rep. 5, 13783 (2015).

${ }^{66}$ J.-P. Peng, J.-Q. Guan, H.-M. Zhang, C.-L. Song, L. Wang, K. He, Q.-K. Xue, and X.-C. Ma, Phys. Rev. B 91, 121113 (2015).

${ }^{67}$ H. D. Ozaydin, H. Sahin, J. Kang, F. M. Peeters, and R. T. Senger, 2D Mater. 2, 044002 (2015)

${ }^{68}$ X. Meng, A. Pant, H. Cai, J. Kang, H. Sahin, B. Chen, K. Wu, S. Yang, A. Suslu, F. M. Peeters, and S. Tongay, Nanoscale 7, 17109 (2015).

${ }^{69}$ H. Cai, J. Kang, H. Sahin, B. Chen, A. Suslu, K. Wu, F. Peeters, X. Meng, and S. Tongay, Nanotechnology 27, 065203 (2016).

${ }^{70}$ B.-J. Wang, X.-H. Li, L.-W. Zhang, G.-D. Wang, and S.-H. Ke, Chin. Phys. B 25, 107101 (2016).

${ }^{71}$ Y. Zhou, S. Li, W. Zhou, X. Zu, and F. Gao, Sci. Rep. 4, 5773 (2014).

${ }^{72}$ L. Wang, R. Chen, Z.-F. Ren, C.-W. Ge, Z.-X. Liu, S.-J. He, Y.-Q. Yu, C.-Y. Wu, and L.-B. Luo, Nanotechnology 27, 215202 (2016).

${ }^{73}$ X. Zhang, X. Zhang, X. Zhang, Y. Zhang, L. Bian, Y. Wu, C. Xie, Y. Han, Y. Wang, P. Gao, L. Wang, and J. Jie, J. Mater. Chem. 22, 22873 (2012).
${ }^{74}$ Y. Jiang, X.-M. Meng, W.-C. Yiu, J. Liu, J.-X. Ding, C.-S. Lee, and S.-T. Lee, J. Phys. Chem. B 108, 2784 (2004).

${ }^{75}$ L. Zhao, Q. Pang, Y. Cai, N. Wang, W. Ge, J. Wang, and S. Yang, J. Phys. D: Appl. Phys. 40, 3587 (2007).

${ }^{76}$ L. Li, Z. Chen, Y. Hu, X. Wang, T. Zhang, W. Chen, and Q. Wang, J. Am. Chem. Soc. 135, 1213 (2013).

${ }^{77}$ Y. Huang, C. Ling, H. Liu, and S. Wang, RSC Adv. 4, 6933 (2014).

${ }^{78}$ K. Tyagi, K. Waters, G. Wang, B. Gahtori, D. Haranath, and R. Pandey, Mater. Res. Express 3, 035013 (2016).

${ }^{79}$ J. O. Island, M. Buscema, M. Barawi, J. M. Clamagirand, J. R. Ares, C. Sanchez, I. J. Ferrer, G. A. Steele, H. S. J. van der Zant, and A. Castellanos-Gomez, Adv. Opt. Mater. 2, 641 (2014).

${ }^{80}$ A. Lipatov, P. M. Wilson, M. Shekhirev, J. D. Teeter, R. Netusil, and A. Sinitskii, Nanoscale 7, 12291 (2015).

${ }^{81}$ M. Barawi, E. Flores, M. Ponthieu, J. R. Ares, F. Cuevas, F. Leardini, I. J. Ferrer, and C. Sanchez, J. Elect. Eng., USA 3, 24 (2015).

${ }^{82}$ A. S. Pawbake, J. O. Island, E. Flores, J. R. Ares, C. Sanchez, I. J. Ferrer, S. R. Jadkar, H. S. J. van der Zant, A. Castellanos-Gomez, and D. J. Late, ACS Appl. Mater. Interfaces 7, 24185 (2015).

${ }^{83}$ Q. Cui, A. Lipatov, J. S. Wilt, M. Z. Bellus, X. C. Zeng, J. Wu, A. Sinitskii, and H. Zhao, ACS Appl. Mater. Interfaces 8, 18334 (2016).

${ }^{84}$ J. Kang, H. Sahin, H. D. Ozaydin, R. T. Senger, and F. M. Peeters, Phys. Rev. B 92, 075413 (2015).

${ }^{85}$ Y. Jin, X. Li, and J. Yang, Phys. Chem. Chem. Phys. 17, 18665 (2015).

${ }^{86} \mathrm{M}$. Li, J. Dai, and C. Zeng, Nanoscale 7, 15385 (2015).

${ }^{87}$ J. K. Osada, S. Bae, M. Tanaka, H. Raebiger, K. Shudo, and T. Suzuki, J. Phys. Chem. C 120, 4653 (2016).

${ }^{88}$ A. Pant, E. Torun, B. Chen, S. Bhat, X. Fan, K. Wu, D. P. Wright, F. M. Peeters, E. Soignard, H. Sahin, and S. Tongay, Nanoscale 8, 16259 (2016).

${ }^{89}$ P. M. Das, G. Danda, A. Cupo, W. M. Parkin, L. Liang, N. Kharche, X. Ling, S. Huang, M. S. Dresselhaus, V. Meunier, and M. Drndic, ACS Nano 10, 5687 (2016).

${ }^{90}$ J. Zhang, H. J. Liu, L. Cheng, J. Wei, J. H. Liang, D. D. Fan, J. Shi, X. F. Tang, and Q. J. Zhang, Sci. Rep. 4, 6452 (2014).

${ }^{91}$ B. Ding, W. Chen, Z. Tang, and J. Zhang, J. Phys. Chem. C 120, 2149 (2016).

${ }^{92}$ Z. Liu and K. Aydin, Nano Lett. 16, 3457 (2016).

${ }^{93}$ V. Sorkin and Y. W. Zhang, 2D Mater. 2, 035007 (2015).

${ }^{94}$ S. Cahangirov, M. Topsakal, E. Akturk, H. Sahin, and S. Ciraci, Phys. Rev. B 102, 236804 (2009).

${ }^{95}$ P. Vogt, P. D. Padova, C. Quaresima, J. Avila, E. Frantzeskakis, M. C. Asensio, A. Resta, B. Ealet, and G. L. Lay, Phys. Rev. Lett. 108, 155501 (2012).

${ }^{96}$ C. L. Lin, R. Arafune, K. Kawahara, N. Tsukahara, E. Minamitami, Y. Kim, N. Takagi, and M. Kawai, Appl. Phys. Express 5, 045802 (2012).

${ }^{97}$ A. Fleurence, R. Friedlein, T. Osaki, H. Kawai, Y. Wang, and Y. Y. Takamura, Phys. Rev. Lett. 108, 245501 (2012).

${ }^{98}$ M. E. Davila, L. Xian, S. Cahangirov, A. Rubio, and G. L. Lay, New J. Phys. 16, 095002 (2014).

${ }^{99}$ S. Saxena, R. P. Chaudhary, and S. Shukla, Sci. Rep. 6, 31073 (2016).

${ }^{100}$ P. De Padova, C. Quaresima, C. Ottaviani, P. M. Sheverdyaeva, P. Moras, C. Carbone, D. Topwal, B. Olivieri, A. Kara, H. Oughaddou, B. Aufray, and G. L. Lay, Appl. Phys. Lett. 96, 261905 (2010).

${ }^{101}$ P. De Padova, C. Quaresima, B. Olivieri, P. Perfetti, and G. L. Lay, Appl. Phys. Lett. 98, 081909 (2011).

${ }^{102}$ M. R. Tchalala, H. Enriquez, A. J. Mayne, A. Kara, S. Roth, M. G. Silly, A. Bendounan, F. Sirotti, T. Greber, B. Aufray, G. Dujardin, M. A. Ali, and H. Oughaddou, Appl. Phys. Lett. 102, 083107 (2013).

${ }^{103}$ P. De Padova, O. Kubo, B. Olivieri, C. Quaresima, T. Nakayama, M. Aono, and G. L. Lay, Nano Lett. 12, 5500 (2012).

${ }^{104}$ B. Feng, H. Li, S. Meng, L. Chen, and K. Wu, Surf. Sci. 645, 74 (2016).

${ }^{105}$ M. Topsakal and S. Ciraci, Phys. Rev. B 81, 024107 (2010).

${ }^{106}$ Y. Jing, Y. Sun, H. Niu, and J. Shen, Phys. Status Solidi B 250, 1505 (2013).

${ }^{107}$ Y. C. Zhao and J. Ni, Phys. Chem. Chem. Phys. 16, 15477 (2014).

${ }^{108}$ Y.-L. Song, Y. Zhang, J.-M. Zhang, and D.-B. Lu, Appl. Surf. Sci. 256, $6313(2010)$

${ }^{109}$ H.-H. Fu, D.-D. Wu, Z.-Q. Zhang, and L. Gu, Sci. Rep. 5, 10547 (2015).

${ }^{110}$ X.-T. An, Y.-Y. Zhang, J.-J. Liu, and S.-S. Li, New J. Phys. 14, 083039 (2012).

${ }^{111}$ T. Saari, C.-Y. Huang, J. Nieminen, W.-F. Tsai, H. Lin, and A. Bansil, Appl. Phys. Lett. 104, 173104 (2014). 
${ }^{112}$ K. Shakouri, H. Simchi, M. Esmaeilzadeh, H. Mazidabadi, and F. M. Peeters, Phys. Rev. B 92, 035413 (2015).

${ }^{113}$ Y. Ding and J. Ni, Appl. Phys. Lett. 95, 083115 (2009).

${ }^{114}$ M. Zare, F. Parhizgar, and R. Asgari, Phys. Rev. B 94, 045443 (2016).

${ }^{115}$ M. Ezawa and N. Nagaosa, Phys. Rev. B 88, 121401 (2013).

${ }^{116}$ Q. Pang, Y. Zhang, J.-M. Zhang, V. Ji, and K.-W. Xu, Nanoscale 3, 4330 (2011)

${ }^{117}$ A. H. Bayani, D. Dideban, and N. Moezi, J. Comput. Electron. 15, 381 (2016).

${ }^{118}$ Y. Wang, J. Zheng, Z. Ni, R. Fei, Q. Liu, R. Quhe, C. Xu, J. Zhou, Z. Gao, and J. Lu, Nano 7, 1250037 (2012).

${ }^{119}$ F.-F. Zhu, W.-J. Chen, Y. Xu, C.-L. Gao, D.-D. Guan, D. Qian, S.-C. Zhang, and J. F. Jia, Nat. Mater. 14, 1020 (2015).

${ }^{120}$ W. Xiong, C. Xia, Y. Peng, J. Du, T. Wang, J. Zhang, and Y. Jia, Phys. Chem. Chem. Phys. 18, 6534 (2016).

${ }^{121}$ B. van den Broek, M. Houssa, G. Pourtois, V. V. Afanasev, and A. Stesmans, Phys. Status Solidi RRL 8, 931 (2014).

${ }^{122}$ B. van den Broek, M. Houssa, K. Iordanidou, G. Pourtois, V. V. Afanasev, and A. Stesmans, 2D Mater. 3, 015001 (2016).

${ }^{123}$ A. Hattori, K. Yada, Y. Tanaka, S. Tanaya, Y. Hatsugai, M. Araidai, M. Sato, and K. Shiraishi, e-print arXiv:1604.04717 [cond-mat.mes-hall].

${ }^{124}$ K. S. Novoselov, D. Jiang, F. Schedin, T. Booth, V. V. Khotkevich, S. Morozov, and A. K. Geim, Proc. Natl. Acad. Sci. U. S. A. 102, 10451 (2005).

${ }^{125}$ Z.-G. Chen, J. Zou, G. Liu, F. Li, Y. Wang, L. Wang, X.-L. Yuan, T. Sekiguchi, H.-M. Cheng, and G. Q. Lu, ACS Nano 2, 2183 (2008).

${ }^{126}$ H. Zeng, C. Zhi, Z. Zhang, X. Wei, X. Wang, W. Guo, Y. Bando, and D. Golberg, Nano Lett. 10, 5049 (2010).

${ }^{127}$ X. Wei, M.-S. Wang, Y. Bando, and D. Golberg, ACS Nano 5, 2916 (2011).

${ }^{128}$ D. K. Samarakon and X.-Q. Wang, Appl. Phys. Lett. 100, 103107 (2012).
${ }^{129}$ M. Topsakal, E. Akturk, and S. Ciraci, Phys. Rev. B 79, 115442 (2009).

${ }^{130}$ A. Lopez-Bezanilla, J. Huang, H. Terrones, and B. G. Sumpter, Nano Lett. 11, 3267 (2011).

${ }^{131}$ J. Zeng, K.-Q. Chen, and C. Q. Sun, Phys. Chem. Chem. Phys. 14, 8032 (2012).

${ }^{132}$ X. Li, X. Wu, X. C. Zeng, and J. Yang, ACS Nano 6, 4104 (2012).

${ }^{133}$ J. Qi, X. Qian, L. Qi, J. Feng, D. Shi, and J. Li, Nano Lett. 12, 1224 (2012).

${ }^{134}$ J. Zhang, Y. Zhang, J.-M. Zhang, and K.-W. Wu, Comput. Theor. Chem. 967, 113 (2011).

${ }^{135}$ A. J. Du, Z. H. Zhu, Y. Chen, G. Q. Lu, and S. C. Smith, Chem. Phys. Lett. 469, 183 (2009).

${ }^{136}$ A. Lopez-Bezanilla, P. Ganesh, P. R. C. Kent, and B. G. Sumpter, Nano Res. 7, 63 (2014).

${ }^{137}$ Q.-L. Rao, Y.-X. Wang, Z. Chen, X.-j. Du, and T.-T. Sun, Superlattices Microstruct. 84, 36 (2015).

${ }^{138}$ Y.-Y. Qi, Y. Zhang, J.-M. Zhang, V. Ji, and K.-W. Xu, Comput. Theor. Chem. 974, 151 (2011).

${ }^{139}$ H. Sahin, S. Cahangirov, M. Topsakal, E. Bekaroglu, E. Akturk, R. T. Senger, and S. Ciraci, Phys. Rev. B 80, 155453 (2009).

${ }^{140}$ Q. Chen, R. Song, C. Chen, and X. Chen, Solid State Commun. 172, 24 (2013).

${ }^{141}$ Y. Dai, X. Chen, and C. Jiang, Physica B 407, 515 (2012).

${ }^{142}$ R. Chandiramouli, Mater. Sci. Semicond. Process. 35, 109 (2015).

${ }^{143}$ G. S. Randhawa, G. Pawa, and S. Kaur, Int. J. Comput. Sci. Eng. 6, 7444 (2016).

${ }^{144}$ G. Seol and J. Guo, Appl. Phys. Lett. 98, 143107 (2011).

${ }^{145}$ J. He, K.-Q. Chen, Z.-Q. Fan, L.-M. Tang, and W. P. Hu, Appl. Phys. Lett. 97, 193305 (2010).

${ }^{146}$ J. Kang, H. Sahin, and F. M. Peeters, J. Phys. Chem. C 119, 9580 (2015).

${ }^{147}$ Z. Zhang, Y. Xie, Q. Peng, and Y. Chen, Sci. Rep. 6, 21639 (2016). 